\title{
TG, DTA Pyrolytic Analysis of Cobalt, Nickel, Copper, Zinc, and 5,8-Dihydroxy-1,4-Naphthoquinone Chelate Complexes
}

\author{
M. Amin Mir $\mathbb{D}^{1}$ and Mohammad Waqar Ashraf $\mathbb{D}^{2}$ \\ ${ }^{1}$ Department of Mathematics \& Natural Sciences, Prince Mohammad Bin Fahd University, AlKhobar, Saudi Arabia \\ ${ }^{2}$ Dean Department of Mathematics \& Natural Sciences, Prince Mohammad Bin Fahd University, AlKhobar, Saudi Arabia \\ Correspondence should be addressed to M. Amin Mir; mohdaminmir@gmail.com
}

Received 27 October 2020; Revised 22 March 2021; Accepted 8 April 2021; Published 4 May 2021

Academic Editor: Pasquale Longo

Copyright (c) 2021 M. Amin Mir and Mohammad Waqar Ashraf. This is an open access article distributed under the Creative Commons Attribution License, which permits unrestricted use, distribution, and reproduction in any medium, provided the original work is properly cited.

\begin{abstract}
The solid state reactions identified on the TG traces with correspondence to DTG peaks consequent to the nonisothermal decomposition of polymetallic chelates of the naphthazarin with $\mathrm{Zn}$ (II), Co (II), Ni (II), and Cu (II) over the temperature range ambient at $800^{\circ} \mathrm{C}$ have been studied kinetically following the Dave and Chopra method as these solid state reactions exhibited their resemblance with the Freeman recommended reaction for kinetic studies. The solid state reactions as described followed first order kinetics. The kinetic data showed the very low value of $Z$ for each of the solid state reaction in reference, concluding on the solid state reactions (the nonisothermal decomposition of polymetallic chelate of $\mathrm{Zn}$ (II), Co (II), Ni (II), and Cu (II) as slow reactions).
\end{abstract}

\section{Introduction}

Due to automation in the recent times, the instruments have become capable of self-operation, improving both accuracy and precision of measurements, as well as relinquishing both investigator's time and patience.

Besides other instruments, the thermal methods provide today the means of solving existing chemical as well as creating new ones. These methods can provide rapid information concerning the thermal stability, composition of pyrolysis intermediates, and composition of the final product as a compound is heated to elevated temperature.

Borrel and Paris [1] carried out the synthesis and stoichiometry of some metal oxinate complexes and their associated thermal stability by using thermogravimetric analysis. The effect of alpha methyl substitution on the oxine ligand in $\mathrm{Cu}$ (II) and $\mathrm{Zn}$ (II) complexes [2] over the solubility products of $\mathrm{Cu}(\mathrm{II})$ and $\mathrm{Zn}$ (II) oxinates and methyloxinates was studied by potentiometric neutralization of acid solutions containing oxine or methyloxine and metallic cations [3]. The thermal stability and volatilization on vacuum of metallic chelates which are derivatives of 8- hydroxyquinoline have been studied by Charles and Langer [4]. Also, it had been observed that the temperature range of volatilization depends on metallic ion electronegativity for the divalent metal 8-hydroxyquinolinates. The thermal stability analysis of these complexes was studied by Wendlandt and Horton [5] using differential thermal analysis (DTA). These 8-hydroxyquinolinates hydrates were also studied by Gore and Wendlandt [6] by using thermogravimetry, differential scanning calorimetry, and reflectance spectra.

The crystalline structures study of this kind of complex [7-9] notifies monoclinic in the $\mathrm{a}$ and $\mathrm{b}$ forms for the copper (II) complexes regardless of the hydration degree. Zn (II) and Cd (II) complexes $[10,11]$ have been found to be monoclinic, but it had been found that these structures depend on the hydration. The zinc (II) and cadmium (II) hydrated complexes show the same b form found for the copper (II) complexes. Several studies have indicated characteristic IR bands for these compounds [12-18]. An attractive group of natural 1,4-naphthoquinones is spinochrome, i.e., the pigments of echinoderms with naphthazarin 1 (5,8-dihydroxy-1,4-naphthoquinone) core 
[19-22]. Naphthoquinones in association with various metals have many medicinal properties [23-26].

The chelating agents are capable of chelating the metal cations having 2-4 valencies in line with the ligancy of metals involved, but the hydroxynaphthoquinone, namely, 5,8-dihydroxy-1,4-naphthoquinone (naphthazarin), a synthetic hydroxynaphthoquinone, appears to be able to form polymetallic chelates with different metal cations due to the presence of an additional hydroxyl group at carbon 8 as compared to its family member: 5-hydroxy-1,4-naphthoquinone (juglone) with the capability to chelate two metal cations initially forming the two six member rings with the progression of more six member ring formation in line with the metal ligancy involving either ligand molecule(s) or coordinated water molecules as the satisfaction of metal ligancy a prerequisite in the chelation process.

The literature survey has provided information on the little work performed so far on the chelating properties of naphthazarin molecule (Scheme 1).

This work centers around the synthesis of polymetallic chelates of different metal cations ( $\mathrm{Zn}$ (II) $\mathrm{Co}(\mathrm{II}), \mathrm{Ni}(\mathrm{II})$, and $\mathrm{Cu}(\mathrm{II})$ ) with naphthazarin and their pyrolysis mapping with emphasis on the detection of stability and instability zones, composition of the pyrolysis intermediates and the synthetic metal chelates, as well, and the kinetics of the nonisothermal decomposition of the polymetallic chelates involving the decomposition reactions detected on the pyrolysis traces with agreement to the type of reaction.

$\mathrm{A}(\mathrm{s}) \longrightarrow \mathrm{B}(\mathrm{s})+\mathrm{C}(\mathrm{g})$.

It was recommended by Freeman and Carrol [27] for the study of the kinetics of the reaction. The Dave and Chopra procedure [28] was applied to study the nonisothermal decomposition reactions kinetically.

\section{Materials and Methods}

The chemicals of high purity were used in the study of chelation of metal cations and naphthazarin at $\mathrm{pH}$ 6. For the synthesis of metal chelates, equal molar of aqueous metal salt solution and ethanolic solution of naphthazarin were mixed and buffered at $\mathrm{pH}$ 6. The resultant mixture was allowed to stand for a period of at least ten days. The crystals so formed were filtered, washed with double distilled water, and shade dried and finally bottled. The process of preparation of chelate complexes was carried out without using any external catalyst. The process takes place via the autocatalytic mechanism.

For pyrolysis mapping, the dried solid mass of metal chelate of naphthazarin with metal cations $\mathrm{Zn}$ (II)/Co (II)/ $\mathrm{Ni}$ (II) $/ \mathrm{Cu}$ (II) was subjected to thermal analysis in nitrogen environment $\left(100 / 200 \mathrm{ml} \mathrm{min}{ }^{-1}\right)$ at $10^{\circ} \mathrm{C} \mathrm{min}{ }^{-1}$ over temperature range ambient at $800^{\circ} \mathrm{C}$. The thermal database is given in Tables 1-4, whereas the pyrolysis traces of different metal cations are shown in Figures 1-6.

2.1. Kinetics of Nonisothermal Decomposition Reaction (Dave and Chopra Method). Dave and Chopra [28] gave the accompanying expression to study the kinetics of

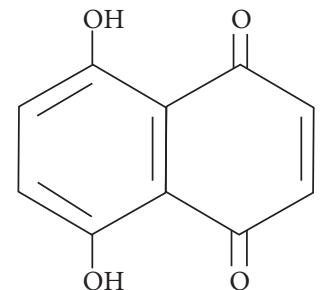

Scheme 1: The systematic representation of naphthoquinone.

nonisothermal decomposition reactions matching the solid state reaction of the type. $\mathrm{A}(\mathrm{s}) \longrightarrow \mathrm{B}(\mathrm{s})+\mathrm{C}(\mathrm{g})$.

$$
k=\frac{(A / m o)^{2 n-1}(-\mathrm{d} x / \mathrm{d} t)}{(A-a)^{2}},
$$

where $k$ is the specific rate constant, $A$ is the total area under the DTG curve, $a$ is the area at time $t, n$ is the order of reaction, $-\mathrm{d} x / \mathrm{d} t$ is the deviation from baseline $(-\mathrm{d} x / \mathrm{d} t=0)$.

For, $n=1$, equation (1) reduces to

$$
K=\frac{\mathrm{d} x / \mathrm{d} t}{A-a} .
$$

Equation (2) together with Arrhenius equation (3)

$$
k=Z e^{-E / R T},
$$

where $Z$ is the frequency factor and gives

$$
\log 10 \mathrm{k}=\log 10 Z-\frac{E}{2.303 R T} .
$$

A straight line relationship is obtained on plotting $\log _{10} k$ against $T^{-1}$, giving intercept as $\log _{10} Z$ and slope $(\tan \theta)$ as $E /$ $2.303 R$ (Figure 3).

The samples of polymetallic chelates of naphthazarin with $\mathrm{Zn}$ (II) or Co (II) or $\mathrm{Ni}$ (II) or $\mathrm{Cu}$ (II) cations were run on EXSTAR TG/DTA 6300 in nitrogen $(100 / 200 \mathrm{ml} / \mathrm{min})$ atmosphere with reference weight of $10.500 \mathrm{mg}$ and reference name alumina powder and temperature program as

$$
\begin{array}{ccccc}
\text { Cal } & \text { Cal } & \text { Cal } / \text { min } & \text { min } & \text { S } \\
31 & 800 & 10 & 0 & 0.5
\end{array}
$$

The sample weights ranging from $1 \mathrm{mg}$ to $3 \mathrm{mg}$ were employed. The small sample size within the limits of sensitivity of balance was so used as to ensure that the heating rate $\left(10^{\circ} \mathrm{C} / \mathrm{min}\right)$ could not depart from its constant value.

The polymetallic chelates of naphthazarin with $\mathrm{Zn}$ (II), Co (II), Ni (II), and Cu (II) were mapped thermally, structurally, and compositionally employing the thermal database generated with the use of instrument "EXSTAR TG/DTA 6300."

\section{Results and Discussion}

The nonisothermal decomposition reactions with correspondence to the well-defined sigmoids on TG traces had been kinetically studied employing the peaks on DTG trace with complete correspondence to sigmoids on TG traces. 
TABLe 1: Analytical data on nonisothermal decomposition of $\left[\left(\mathrm{C}_{10} \mathrm{H}_{6} \mathrm{O}_{4}\right)_{3}(\mathrm{Zn})_{2}\right] 2 \frac{1}{2} \mathrm{H}_{2} \mathrm{O}$.

\begin{tabular}{|c|c|c|c|c|}
\hline Reaction & Loss & Composition & Found & Calc. \\
\hline $\begin{array}{l}\text { Plateau I, ambient at } 24.1^{\circ} \mathrm{C} \\
\text { Sigmoid I } \\
24.1^{\circ} \mathrm{C}-100^{\circ} \mathrm{C}\end{array}$ & ${ }_{21 / 2}-$ & $\left(\mathrm{C}_{10} \mathrm{H}_{6} \mathrm{O}_{4}\right)_{3}(\mathrm{Zn})_{2} 2 \frac{1}{2} \mathrm{H}_{2} \mathrm{O}$ & - & - \\
\hline $\mathrm{T}_{\mathrm{i}}^{\circ} \mathrm{C}$ & & & 24.1 & - \\
\hline $\begin{array}{l}\mathrm{T}_{\mathrm{f}}{ }^{\circ} \mathrm{C} \\
\text { Loss to }\end{array}$ & & & 100 & - \\
\hline $\begin{array}{c}\left(\mathrm{C}_{10} \mathrm{H}_{6} \mathrm{O}_{4}\right)_{3}(\mathrm{Zn})_{2} \\
\text { Plateau II, } 100^{\circ}-150^{\circ} \mathrm{C}\end{array}$ & & $\left(\mathrm{C}_{10} \mathrm{H}_{6} \mathrm{O}_{4}\right)_{3}(\mathrm{Zn})_{2}$ & 6.30 & 6.37 \\
\hline $\begin{array}{l}\text { Sigmoid II } \\
150^{\circ}-200^{\circ} \mathrm{C}\end{array}$ & $1 / 2 \mathrm{C}_{10} \mathrm{H}_{6} \mathrm{O}_{4}$ & & & \\
\hline $\mathrm{T}_{\mathrm{i}}^{\circ} \mathrm{C}$ & & & 150 & \\
\hline $\begin{array}{l}\mathrm{T}_{\mathrm{f}}{ }^{\circ} \mathrm{C} \\
\text { Loss to }\end{array}$ & & & 200 & \\
\hline $5 / 2\left(\mathrm{C}_{10} \mathrm{H}_{6} \mathrm{O}_{4}\right)(\mathrm{Zn})_{2}$ & & & 17.80 & 18.12 \\
\hline $\begin{array}{l}\text { Plateau III, } 200^{\circ}-500^{\circ} \mathrm{C} \\
\text { Sigmoid III } \\
500^{\circ}-529^{\circ} \mathrm{C}\end{array}$ & & $5 / 2\left(\mathrm{C}_{10} \mathrm{H}_{6} \mathrm{O}_{4}\right)(\mathrm{Zn})_{2}$ & & \\
\hline $\mathrm{T}_{\mathrm{i}}{ }^{\circ} \mathrm{C}$ & & & 500 & \\
\hline $\mathrm{T}_{\mathrm{f}}^{\circ} \mathrm{C}$ & & & 529 & \\
\hline $\begin{array}{l}\text { Loss to } \\
\left(\mathrm{C}_{10} \mathrm{H}_{6} \mathrm{O}_{4}\right)_{2}(\mathrm{Zn})_{2}\end{array}$ & $1 / 2 \mathrm{C}_{10} \mathrm{H}_{6} \mathrm{O}_{4}$ & & 29.90 & 31.54 \\
\hline $\begin{array}{l}\text { Plateau IV, } 529^{\circ}-600^{\circ} \mathrm{C} \\
\text { Sigmoid IV } \\
600^{\circ}-700^{\circ} \mathrm{C}\end{array}$ & ${ }^{\mathrm{C}} 10^{\mathrm{H}} 6^{\mathrm{O}} 4$ & $\left(\mathrm{C}_{10} \mathrm{H}_{6} \mathrm{O}_{4}\right)_{2}(\mathrm{Zn})_{2}$ & & \\
\hline $\mathrm{T}_{\mathrm{i}}^{\circ} \mathrm{C}$ & & & 600 & \\
\hline $\mathrm{T}_{\mathrm{f}}{ }^{\circ} \mathrm{C}$ & & & 700 & \\
\hline $\begin{array}{l}\text { Loss to } \\
\left(\mathrm{C}_{10} \mathrm{H}_{6} \mathrm{O}_{4}\right)(\mathrm{Zn})_{2} \\
700^{\circ} \text { onwards }\end{array}$ & & & 55.76 & 57.05 \\
\hline
\end{tabular}

TABLE 2: Analytical data on the nonisothermal decomposition of $\left[\left(\mathrm{C}_{10} \mathrm{H}_{6} \mathrm{O}_{4}\right)_{3}(\mathrm{Co})_{2} .4 \mathrm{H}_{2} \mathrm{O}\right] 3 \mathrm{H}_{2} \mathrm{O}$.

\begin{tabular}{|c|c|c|c|c|}
\hline Reaction & Loss & Composition & Found & Calc. \\
\hline $\begin{array}{l}\text { Plateau I, ambient at } 100^{\circ} \mathrm{C} \\
\text { Sigmoid I } \\
100^{\circ} \mathrm{C}-169^{\circ} \mathrm{C}\end{array}$ & $7 \overline{\mathrm{H}_{2}} \mathrm{O}$ & {$\left[\left(\mathrm{C}_{10} \mathrm{H}_{6} \mathrm{O}_{4}\right)_{3}(\mathrm{Co})_{2} \cdot 4 \mathrm{H}_{2} \mathrm{O}\right] 3 \mathrm{H}_{2} \mathrm{O}$} & - & - \\
\hline $\mathrm{T}_{\mathrm{i}}{ }^{\circ} \mathrm{C}$ & & & 100 & - \\
\hline $\begin{array}{l}\mathrm{T}_{\mathrm{f}}{ }^{\circ} \mathrm{C} \\
\text { Loss to }\end{array}$ & & & 169 & - \\
\hline $\begin{array}{l}\quad\left(\mathrm{C}_{10} \mathrm{H}_{6} \mathrm{O}_{4}\right)_{3}(\mathrm{Co})_{2} \\
\text { Plateau II, } 169^{\circ}-200^{\circ} \mathrm{C}\end{array}$ & & $\left(\mathrm{C}_{10} \mathrm{H}_{6} \mathrm{O}_{4}\right)_{3}(\mathrm{Co})_{2}$ & 15.17 & 15.46 \\
\hline $\begin{array}{l}\text { Sigmoid II } \\
200^{\circ}-363^{\circ} \mathrm{C}\end{array}$ & $3 / 2 \mathrm{C}_{10} \mathrm{H}_{6} \mathrm{O}_{4}$ & & & \\
\hline $\begin{array}{l}\mathrm{T}_{\mathrm{i}}{ }^{\circ} \mathrm{C} \\
\mathrm{T}_{\mathrm{f}}{ }^{\circ} \mathrm{C} \\
\text { Loss to }\end{array}$ & & & $\begin{array}{l}200 \\
363\end{array}$ & \\
\hline $3 / 2\left(\mathrm{C}_{10} \mathrm{H}_{6} \mathrm{O}_{4}\right)(\mathrm{Co})_{2}$ & & & 49.08 & 49.26 \\
\hline $\begin{array}{l}\text { Plateau III, } 363^{\circ}-500^{\circ} \mathrm{C} \\
\text { Sigmoid III } \\
500^{\circ}-600^{\circ} \mathrm{C}\end{array}$ & $3 / 2 \mathrm{C}_{10} \mathrm{H}_{6} \mathrm{O}_{4}$ & $3 / 2\left(\mathrm{C}_{10} \mathrm{H}_{6} \mathrm{O}_{4}\right)(\mathrm{Co})_{2}$ & & \\
\hline $\begin{array}{l}\mathrm{T}_{\mathrm{i}}{ }^{\circ} \mathrm{C} \\
\mathrm{T}_{\mathrm{f}}^{\circ} \mathrm{C} \\
\text { Loss to }\end{array}$ & & & $\begin{array}{l}500 \\
600\end{array}$ & \\
\hline $\mathrm{Co}_{3} \mathrm{O}_{8}$ & & & 83.56 & 87.78 \\
\hline $\mathrm{Co}_{3} \mathrm{O}_{8}$ & & & 16.44 & 12.19 \\
\hline
\end{tabular}


TABLe 3: Analytical data on the nonisothermal decomposition of $\left[\left(\mathrm{C}_{10} \mathrm{H}_{6} \mathrm{O}_{4}\right)_{3}\left(\mathrm{Ni}_{2}{ }_{2} 4 \mathrm{H}_{2} \mathrm{O}\right] 1 \frac{1}{2} \mathrm{H}_{2} \mathrm{O}\right.$.

\begin{tabular}{|c|c|c|c|c|}
\hline Reaction & Loss & Composition & Found & Calc. \\
\hline Plateau I, ambient at $99.9^{\circ} \mathrm{C}$ & - & {$\left[\left(\mathrm{C}_{10} \mathrm{H}_{6} \mathrm{O}_{4}\right)_{3}(\mathrm{Ni})_{2} \cdot 4 \mathrm{H}_{2} \mathrm{O}\right] \frac{1}{2} \mathrm{H}_{2} \mathrm{O}$} & - & - \\
\hline Sigmoid I & $5 \frac{1}{2} 2 \mathrm{H}_{2} \mathrm{O}$ & & 12.98 & 12.60 \\
\hline \multicolumn{5}{|l|}{$99.9^{\circ}-136^{\circ} \mathrm{C}$} \\
\hline $\mathrm{T}_{\mathrm{i}}{ }^{\circ} \mathrm{C}$ & & & 99.9 & \\
\hline $\mathrm{T}_{\mathrm{f}}{ }^{\circ} \mathrm{C}$ & & & 136 & \\
\hline \multicolumn{5}{|l|}{ Loss to } \\
\hline \multicolumn{5}{|l|}{$\left(\mathrm{C}_{10} \mathrm{H}_{6} \mathrm{O}_{4}\right)_{3}(\mathrm{Ni})_{2}$} \\
\hline \multicolumn{5}{|l|}{ Plateau II, $136^{\circ}-300^{\circ} \mathrm{C}$} \\
\hline Sigmoid II & $11 / 4 \mathrm{C}_{10} \mathrm{H}_{6} \mathrm{O}_{4}$ & & 43.28 & 42.84 \\
\hline \multicolumn{5}{|l|}{$300^{\circ}-365^{\circ} \mathrm{C}$} \\
\hline $\mathrm{T}_{\mathrm{i}}{ }^{\circ} \mathrm{C}$ & & & 300 & \\
\hline $\mathrm{T}_{\mathrm{f}}{ }^{\circ} \mathrm{C}$ & & & 365 & \\
\hline \multicolumn{5}{|l|}{ Loss to } \\
\hline \multicolumn{5}{|l|}{$7 / 4\left(\mathrm{C}_{10} \mathrm{H}_{6} \mathrm{O}_{4}\right)(\mathrm{Ni})_{2}$} \\
\hline Plateau III, $365^{\circ}-500^{\circ} \mathrm{C}$ & & $7 / 4\left(\mathrm{C}_{10} \mathrm{H}_{6} \mathrm{O}_{4}\right)(\mathrm{Ni})_{2}$ & & \\
\hline Sigmoid III & $7 / 4 \mathrm{C}_{10} \mathrm{H}_{6} \mathrm{O}_{4}$ & & 77.73 & 81.17 \\
\hline \multicolumn{5}{|l|}{$500^{\circ}-600^{\circ} \mathrm{C}$} \\
\hline $\mathrm{T}_{\mathrm{i}}^{\circ} \mathrm{C}$ & & & 500 & \\
\hline $\mathrm{T}_{\mathrm{f}}{ }^{\circ} \mathrm{C}$ & & & 600 & \\
\hline \multicolumn{5}{|l|}{ Loss to } \\
\hline \multicolumn{5}{|l|}{$\mathrm{NiO}$} \\
\hline $\mathrm{NiO}\left(600^{\circ} \mathrm{C}\right.$ onwards $)$ & & & 22.27 & 18.83 \\
\hline
\end{tabular}

TABLE 4: Analytical data on the nonisothermal decomposition of $\left[\left(\mathrm{C}_{10} \mathrm{H}_{6} \mathrm{O}_{4}\right)_{3}(\mathrm{Cu})_{2}\right] 4 \mathrm{H}_{2} \mathrm{O}$.

\begin{tabular}{|c|c|c|c|c|}
\hline Reaction & Loss & Composition & Found & Calc \\
\hline $\begin{array}{l}\text { Plateau I ambient at } 26.5^{\circ} \mathrm{C} \\
\text { Sigmoid I } \\
26.5^{\circ} \mathrm{C}-71^{\circ} \mathrm{C} .\end{array}$ & $-\overline{\mathrm{H}_{2} \mathrm{O}}$ & {$\left[\left(\mathrm{C}_{10} \mathrm{H}_{6} \mathrm{O}_{4}\right)_{3}(\mathrm{Cu})_{2}\right] 4 \mathrm{H}_{2} \mathrm{O}$} & - & - \\
\hline $\mathrm{T}_{\mathrm{i}}^{\circ} \mathrm{C}$ & & & 26.5 & - \\
\hline $\mathrm{T}_{\mathrm{f}}^{\circ} \mathrm{C}$ & & & 71 & - \\
\hline $\begin{array}{l}\text { Loss to } \\
\left(\mathrm{C}_{10} \mathrm{H}_{6} \mathrm{O}_{4}\right)_{3}(\mathrm{Cu})_{2}\end{array}$ & & & 9.52 & 9.38 \\
\hline $\begin{array}{l}\text { Plateau II, } 71^{\circ}-300^{\circ} \mathrm{C} \\
\text { Sigmoid II } \\
300^{\circ}-400^{\circ} \mathrm{C}\end{array}$ & $\mathrm{C}_{10} \mathrm{H}_{6} \mathrm{O}_{4}$ & $\left(\mathrm{C}_{10} \mathrm{H}_{6} \mathrm{O}_{4}\right)_{3}(\mathrm{Cu})_{2}$ & & \\
\hline $\begin{array}{l}\mathrm{T}_{\mathrm{i}}{ }^{\circ} \mathrm{C} \\
\mathrm{T}_{\mathrm{f}}{ }^{\circ} \mathrm{C} \\
\text { Loss to }\end{array}$ & & & $\begin{array}{l}300 \\
400\end{array}$ & \\
\hline $\begin{array}{c}\left(\mathrm{C}_{10} \mathrm{H}_{6} \mathrm{O}_{4}\right)_{2}(\mathrm{Cu})_{2} \\
\text { Plateau III, } 400^{\circ}-500^{\circ} \mathrm{C}\end{array}$ & & $\left(\mathrm{C}_{10} \mathrm{H}_{6} \mathrm{O}_{4}\right)_{2}(\mathrm{Cu})_{2}$ & 35.64 & 34.11 \\
\hline $\begin{array}{l}\text { Sigmoid III } \\
500^{\circ}-545^{\circ} \mathrm{C}\end{array}$ & $3 / 2\left(\mathrm{C}_{10} \mathrm{H}_{6} \mathrm{O}_{4}\right)$ & & & \\
\hline $\mathrm{T}_{\mathrm{i}}^{\circ} \mathrm{C}$ & & & 500 & \\
\hline $\begin{array}{l}\mathrm{T}_{\mathrm{f}}^{\circ} \mathrm{C} \\
\text { Loss to }\end{array}$ & & & 545 & \\
\hline $1 / 2 \mathrm{C}_{10} \mathrm{H}_{6} \mathrm{O}_{4} \mathrm{Cu}_{2}$ & & & 68.63 & 70.57 \\
\hline
\end{tabular}

3.1. Polymetallic Chelate of Naphthazarin with Zn (II). The thermal database resulted in the leads on the tentative structural pattern of this polymetallic chelate, consisting 02 moles of $\mathrm{Zn}$ (II) and 03 moles of naphthazarin and $2 \frac{1}{2}$ water molecules as lattice water (Figure 2).

Composition: $\left[\left(\mathrm{C}_{10} \mathrm{H}_{6} \mathrm{O}_{4}\right)_{3}(\mathrm{Zn})_{2}\right] 2 \frac{1}{2} 2 \mathrm{H}_{2} \mathrm{O}$.

3.1.1. Proposed Structure. Initially, the thermal stability of metal chelate $\left[\left(\mathrm{C}_{10} \mathrm{H}_{6} \mathrm{O}_{4}\right)_{3} \quad(\mathrm{Zn})_{2}\right] \quad 2 \frac{1}{2} \quad \mathrm{H}_{2} \mathrm{O}$ exhibited ambient at $24.1^{\circ} \mathrm{C}$ as first plateau temperature range with $24.1^{\circ} \mathrm{C}$ as procedural decomposition temperature (pdt as its acronym). The solid mass began to lose lattice water $2 \frac{1}{2}$ $\mathrm{H}_{2} \mathrm{O}$, and the loss was complete at $100^{\circ} \mathrm{C}$, after which the TG trace levelled off with extension to $150^{\circ} \mathrm{C}$ (second plateau: $100^{\circ} \mathrm{C}-150^{\circ} \mathrm{C}$ ).

With the increase of temperature beyond $150^{\circ} \mathrm{C}$, the intermediate composition of structure $\left(\mathrm{C}_{10} \mathrm{H}_{6} \mathrm{O}_{4}\right)_{3}(\mathrm{Zn})_{2}$ was reduced to $5 / 2\left(\mathrm{C}_{10} \mathrm{H}_{6} \mathrm{O}_{4}\right)(\mathrm{Zn})_{2}$ with the departure of half molecule of $\mathrm{C}_{10} \mathrm{H}_{6} \mathrm{O}_{4}$ in the temperature range $150^{\circ}-200^{\circ} \mathrm{C}$ 


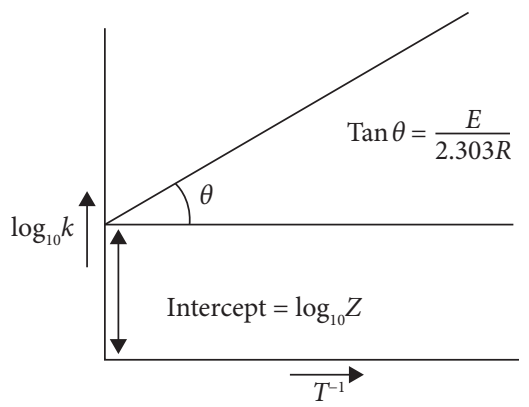

Figure 1: Plot of $\log _{10} k$ against $T^{-1}$.

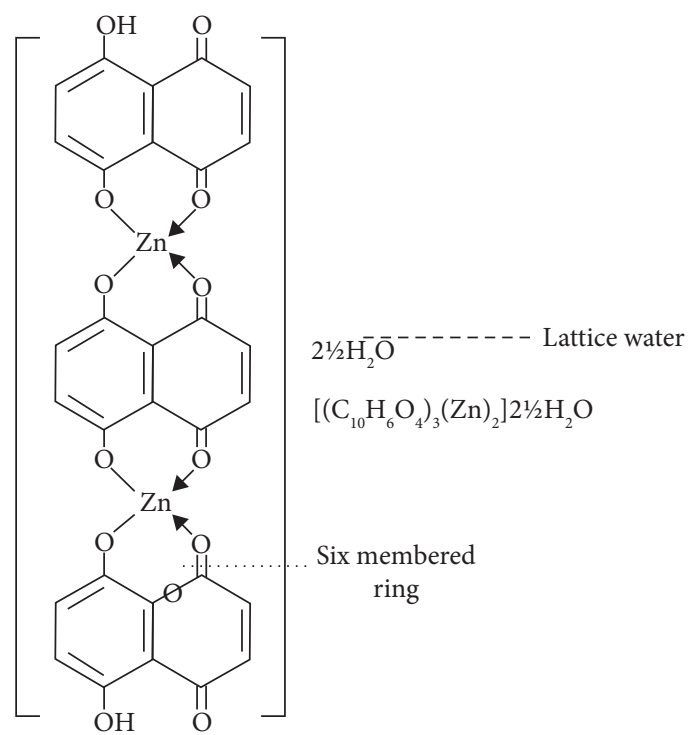

No. of six member rings: 04

Formula weight: 745

Lattice water: $2 \frac{1}{2} \mathrm{H}_{2} \mathrm{O}$

Coordinated water: nil

Zinc metal ligancy: 04

Figure 2: The chelate complex of ligand with zinc.

(second sigmoid). The weight constancy of the second intermediate $\left(5 / 2\left(\mathrm{C}_{10} \mathrm{H}_{6} \mathrm{O}_{4}\right)_{3}(\mathrm{Zn})_{2}\right)$ was exhibited in the temperature $200^{\circ}-500^{\circ} \mathrm{C}$ (third plateau). The second intermediate $5 / 2\left(\mathrm{C}_{10} \mathrm{H}_{6} \mathrm{O}_{4}\right)(\mathrm{Zn})_{2}$ lost further half molecule of $\mathrm{C}_{10} \mathrm{H}_{6} \mathrm{O}_{4}$ in $500^{\circ}-520^{\circ} \mathrm{C}$ temperature range generating still another (third intermediate) intermediate $\left(\mathrm{C}_{10} \mathrm{H}_{6} \mathrm{O}_{4}\right)_{2}(\mathrm{Zn})_{2}$ showing weight constancy from $529^{\circ}$ to $600^{\circ} \mathrm{C}$. With further supply of energy, the $\left(\mathrm{C}_{10} \mathrm{H}_{6} \mathrm{O}_{4}\right)_{2}(\mathrm{Zn})_{2}$, departed a molecule of $\mathrm{C}_{10} \mathrm{H}_{6} \mathrm{O}_{4}$, leaving behind $\left(\mathrm{C}_{10} \mathrm{H}_{6} \mathrm{O}_{4}\right)(\mathrm{Zn})_{2}$ (forth intermediate) showing weight constancy in temperature range of $700^{\circ} \mathrm{C}$ onwards till $800^{\circ} \mathrm{C}$, the other extreme of the temperature range over which the sample was pyrolysed.

The sample could not be further pyrolysed beyond $800^{\circ} \mathrm{C}$ due to the instrumental limitation. It showed that the sample could not be led to the complete combustion level, the stage of complete departure of organic matter from the sample mass, leaving behind $\mathrm{ZnO}$ (zinc oxide).

The possible nonisothermal decomposition reactions identified on TG trace (Figure 6) are described as follows:
(I) $\left[\left(\mathrm{C}_{10} \mathrm{H}_{6} \mathrm{O}_{4}\right)_{3}(\mathrm{Zn})_{2}\right] 2 \mathrm{H}_{2} \mathrm{O}^{24.1^{\circ-100^{\circ} \mathrm{C}}}\left[\left(\mathrm{C}_{10} \mathrm{H}_{6} \mathrm{O}_{4}\right)_{3}\right.$ $\left.(\mathrm{Zn})_{2}\right]+2 \mathrm{H}_{2} \mathrm{O}$

(II) $\left(\mathrm{C}_{10} \mathrm{H}_{6} \mathrm{O}_{4}\right)_{3} \quad(\mathrm{Zn})_{2} \stackrel{150^{\circ}-200^{\circ} \mathrm{C}}{\longrightarrow} 5 / 2 \mathrm{C}_{10} \mathrm{H}_{6} \mathrm{O}_{4} \quad(\mathrm{Zn})_{2}+$ $1 / 2 \mathrm{C}_{10} \mathrm{H}_{6} \mathrm{O}_{4}$

(III) $5 / 4\left(\mathrm{C}_{10} \mathrm{H}_{6} \mathrm{O}_{4}\right)_{3}(\mathrm{Zn})_{2} \stackrel{500^{\circ}-529^{\circ} \mathrm{C}}{\longrightarrow}\left(\mathrm{C}_{10} \mathrm{H}_{6} \mathrm{O}_{4}\right)_{2}(\mathrm{Zn})_{2}+$ $1 / 2 \mathrm{C}_{10} \mathrm{H}_{6} \mathrm{O}_{4}$

(IV) $\left(\mathrm{C}_{10} \mathrm{H}_{6} \mathrm{O}_{4}\right)_{3}(\mathrm{Zn})_{2} \stackrel{600^{\circ}-700^{\circ} \mathrm{C}}{\longrightarrow}\left(\mathrm{C}_{10} \mathrm{H}_{6} \mathrm{O}_{4}\right)_{2}(\mathrm{Zn})_{2}+$ $\mathrm{C}_{10} \mathrm{H}_{6} \mathrm{O}_{4}$

$\left(\mathrm{C}_{10} \mathrm{H}_{6} \mathrm{O}_{4}\right)(\mathrm{Zn})_{2}\left(700^{\circ} \mathrm{C}\right.$ onwards $)$ could not be pyrolysed further due to instrumental limitation.

The analytical data on the pyrolysis journey on the polymetallic chelate of naphthazarin with zinc as shown by TG mapping are given in Table 1.

3.2. Polymetallic Chelate of Naphthazarin with Co (II). Composition: $\left[\left(\mathrm{C}_{10} \mathrm{H}_{6} \mathrm{O}_{4}\right)_{3}(\mathrm{Co})_{2} .4 \mathrm{H}_{2} \mathrm{O}\right] 3 \mathrm{H}_{2} \mathrm{O}$. 


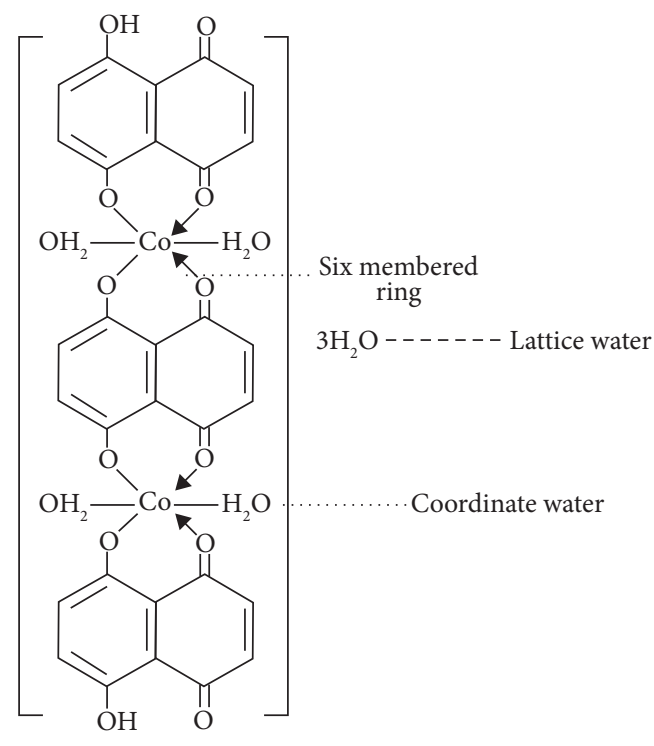

No. of six membered rings: 04

Formula weight: 814

Lattice water: $3 \mathrm{H}_{2} \mathrm{O}$

Coordinator water: $4 \mathrm{H}_{2} \mathrm{O}$

Cobalt ligancy: 06

Figure 3: The chelate complex of ligand with copper.

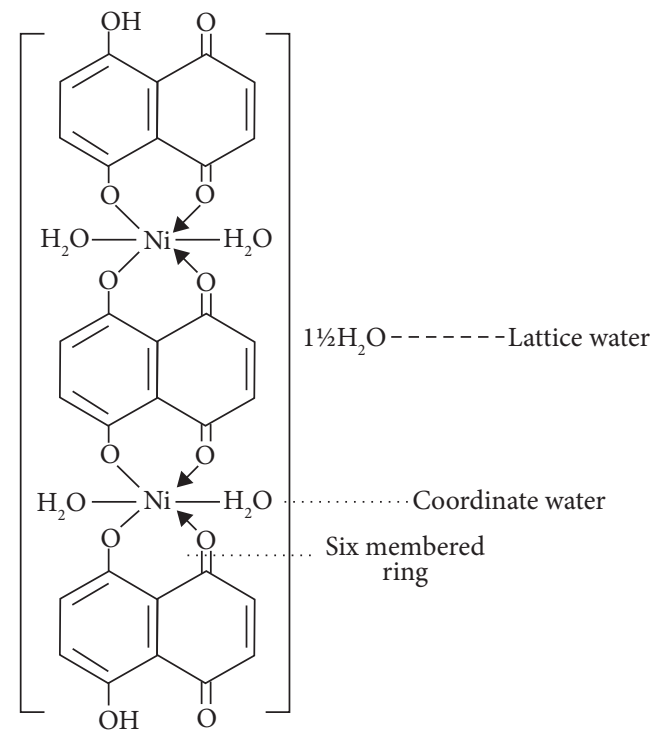

Formula weight: 787

No. of six membered ring: 04

Coordinated water: $04 \mathrm{H}_{2} \mathrm{O}$

Lattice water: $1 \frac{1}{2} \mathrm{H}_{2} \mathrm{O}$

Nickel metal ligancy: 06

Figure 4: The chelate complex of ligand with cobalt.

3.2.1. Proposed Structure. The $\left[\left(\mathrm{C}_{10} \mathrm{H}_{6} \mathrm{O}_{4}\right)_{3}(\mathrm{Co})_{2} \quad 4 \mathrm{H}_{2} \mathrm{O}\right]$ $3 \mathrm{H}_{2} \mathrm{O}$ had been found composing of 02 moles of cobalt cation, 03 moles of $\mathrm{C}_{10} \mathrm{H}_{6} \mathrm{O}_{4}$ (naphthazarin molecules), 04

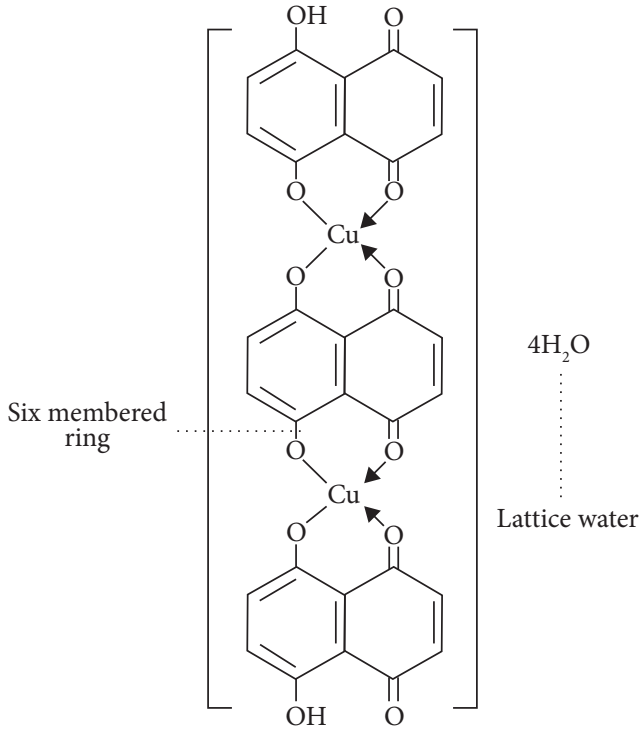

Six membered rings: 04

Formula weight: 768

Lattice water: $4 \mathrm{H}_{2} \mathrm{O}$

Copper metal ligancy: 04

Figure 5: The chelate complex of ligand with nickel.

coordinated water molecules, and 03 lattice water molecules. This polymetallic chelate on its formation under the applied condition composed of additional 04 six membered rings in addition to the 02 six membered rings of the chelating agents (Figure 3).

The nonisothermal decomposition of the thermal mapping spectrum showed the thermal stability at initial states as ambience to $100^{\circ} \mathrm{C}$ (pdt: $100^{\circ} \mathrm{C}$ ). Beyond $100^{\circ} \mathrm{C}$, with the increase of temperature, the structural degeneration occurred, proceeding slowly to $169^{\circ} \mathrm{C}$ with the loss of $7 \mathrm{H}_{2} \mathrm{O}$ including lattice and coordinates water molecules. The TG trace was levelled off between $169^{\circ}$ and $200^{\circ} \mathrm{C}$ showing weight constancy range with correspondence to $\left(\mathrm{C}_{10} \mathrm{H}_{6} \mathrm{O}_{4}\right)_{3}(\mathrm{Co})_{2}$, the intermediate composition. The further incremental increase of temperature beyond $200^{\circ} \mathrm{C}$ registered the further departure of organic matter and stopped at $363^{\circ} \mathrm{C}$, with departure of $1 \frac{1 / 2}{2}\left(\mathrm{C}_{10} \mathrm{H}_{6} \mathrm{O}_{4}\right)$ between $200^{\circ}$ and $363^{\circ} \mathrm{C}$. The plateau $363^{\circ}-500^{\circ} \mathrm{C}$ had a match with another intermediate composition, $1 \frac{1}{2}\left(\mathrm{C}_{10} \mathrm{H}_{6} \mathrm{O}_{4}\right)(\mathrm{Co})_{2}$. This intermediate $1 \frac{1}{2}\left(\mathrm{C}_{10} \mathrm{H}_{6} \mathrm{O}_{4}\right)(\mathrm{Co})_{2}$ lost, with the rise of temperature slowly to $600^{\circ} \mathrm{C}$, the organic residue $1 \frac{1}{2}\left(\mathrm{C}_{10} \mathrm{H}_{6} \mathrm{O}_{4}\right)$, leaving behind $\mathrm{Co}_{3} \mathrm{O}_{8}$. The calculated data on the periodic losses covering thermal spectrum had a close agreement to the experimental data. The data compilation is given in Table 2.

The thermal spectrum recorded on $\left[\left(\mathrm{C}_{10} \mathrm{H}_{6} \mathrm{O}_{4}\right)_{2}(\mathrm{Co})_{2}\right.$ $\left.4 \mathrm{H}_{2} \mathrm{O}\right] 3 \mathrm{H}_{2} \mathrm{O}$ exhibited 04 plateaus, the constant weight zones, and 03 sigmoids $\left(100^{\circ}-169^{\circ} \mathrm{C}, 200^{\circ}-363^{\circ} \mathrm{C}\right.$, and $\left.500^{\circ}-600^{\circ} \mathrm{C}\right)$. The sigmoids on TG trace had corresponding peaks recorded on DTG (Figure 7). 


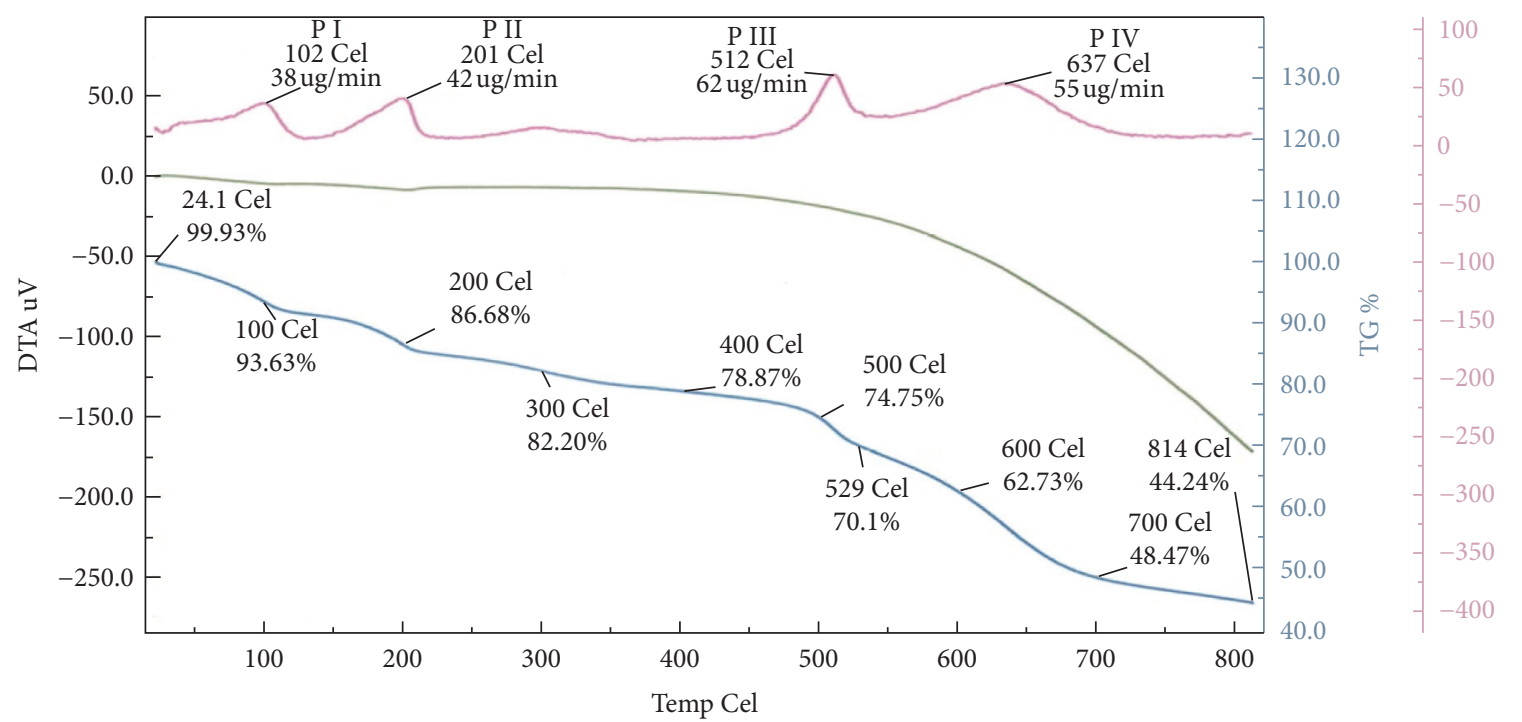

FIGURE 6: Simultaneous pyrolysis mapping of polymetallic chelates of zinc with naphthazarin TG/DTA.

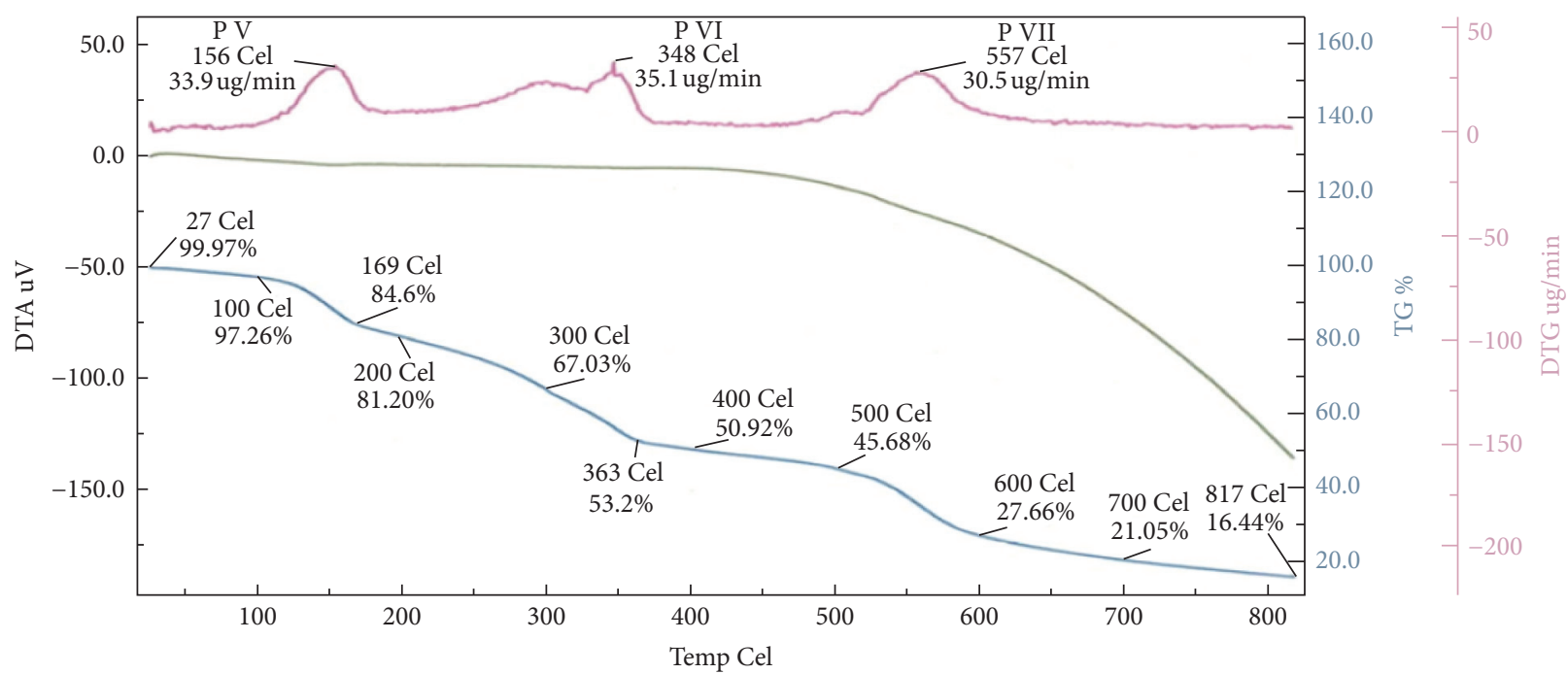

FIGURE 7: Simultaneous pyrolysis mapping of polymetallic chelates of cobalt with naphthazarin TG/DTA.

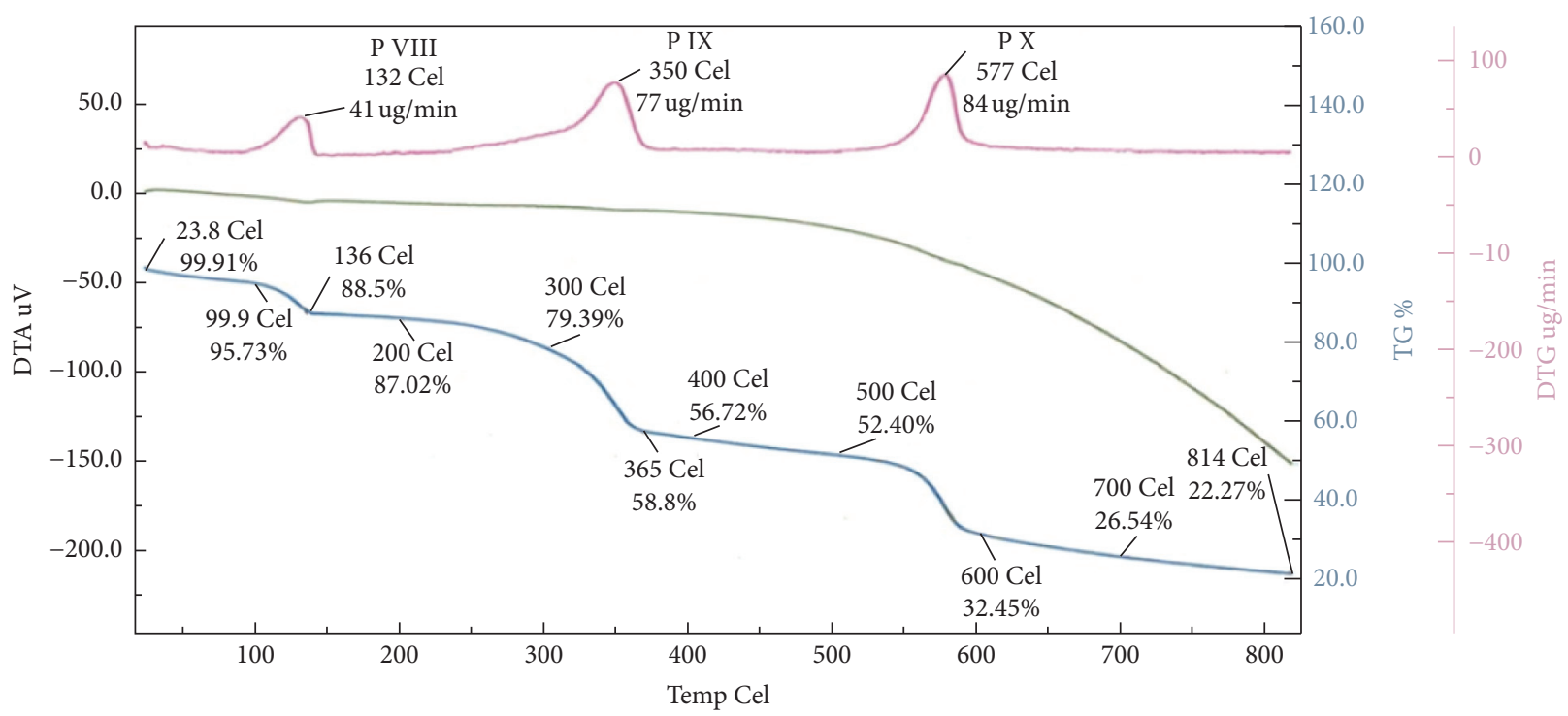

FIGURE 8: Simultaneous pyrolysis mapping of polymetallic chelates of nickel with naphthazarin TG/DTA. 


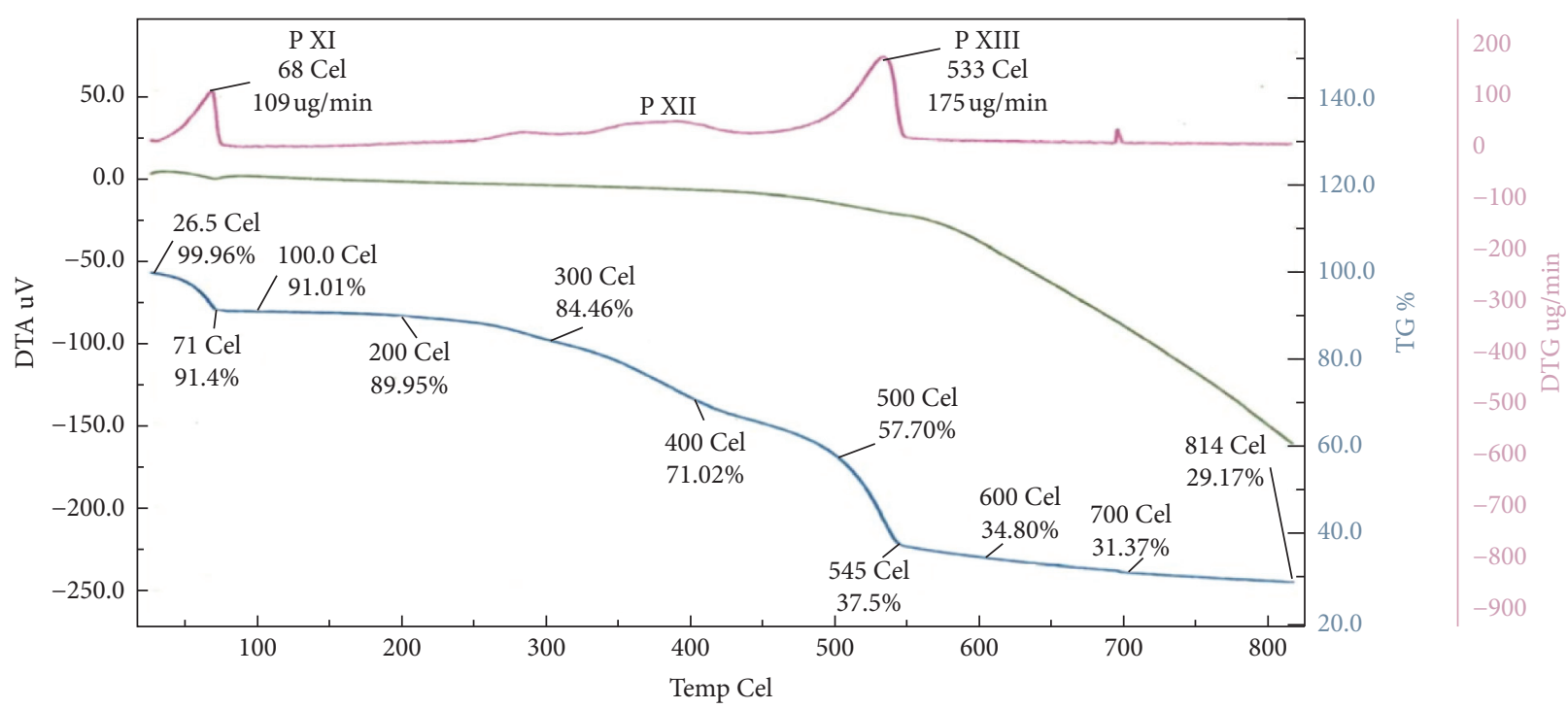

Figure 9: Simultaneous pyrolysis mapping of polymetallic chelates of copper with naphthazarin TG/DTA. Rxn: $\left[\left(\mathrm{C}_{10} \mathrm{H}_{6} \mathrm{O}_{4}\right)_{3}(\mathrm{Zn})_{2}\right] 21 / 2 \mathrm{H}_{2} \mathrm{O} \stackrel{24.1^{\circ}-100^{\circ} \mathrm{C}}{\longrightarrow}\left[\left(\mathrm{C}_{10} \mathrm{H}_{6} \mathrm{O}_{4}\right)_{3}(\mathrm{Zn})_{2}\right]$. Rxn: $\left[\left(\mathrm{C}_{10} \mathrm{H}_{6} \mathrm{O}_{4}\right)_{3}(\mathrm{Zn})_{2}\right] \stackrel{150^{\circ}-200^{\circ} \mathrm{C}}{\longrightarrow} 5 / 2 \mathrm{C}_{10} \mathrm{H}_{6} \mathrm{O}_{4}\left(\mathrm{Zn}_{2}+1 / 2 \mathrm{C}_{10} \mathrm{H}_{6} \mathrm{O}_{4}\right.$.

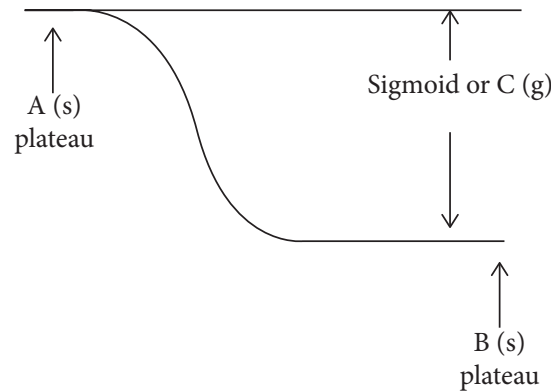

Scheme 2: Graphic representation of solid state reaction $\mathrm{A}(\mathrm{s}) \longrightarrow \mathrm{B}(\mathrm{s})+\mathrm{C}(\mathrm{g}) \uparrow$.

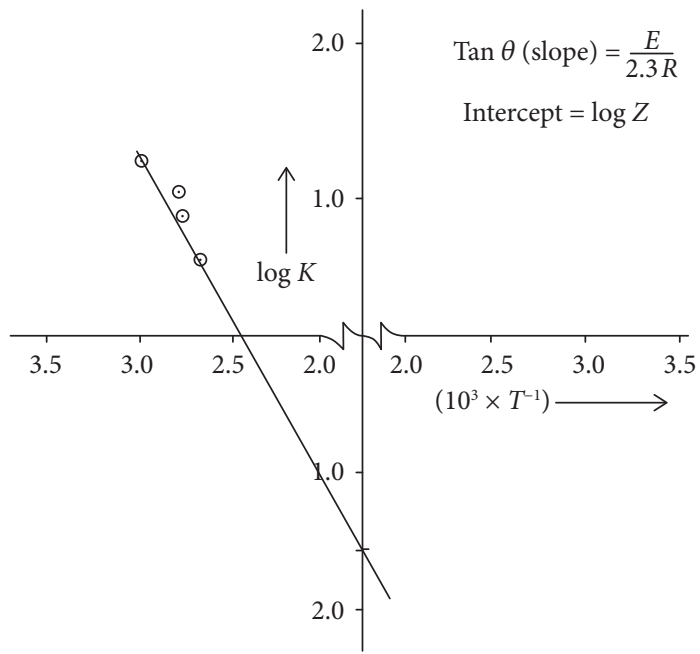

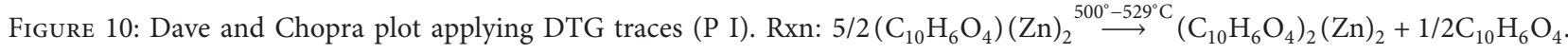




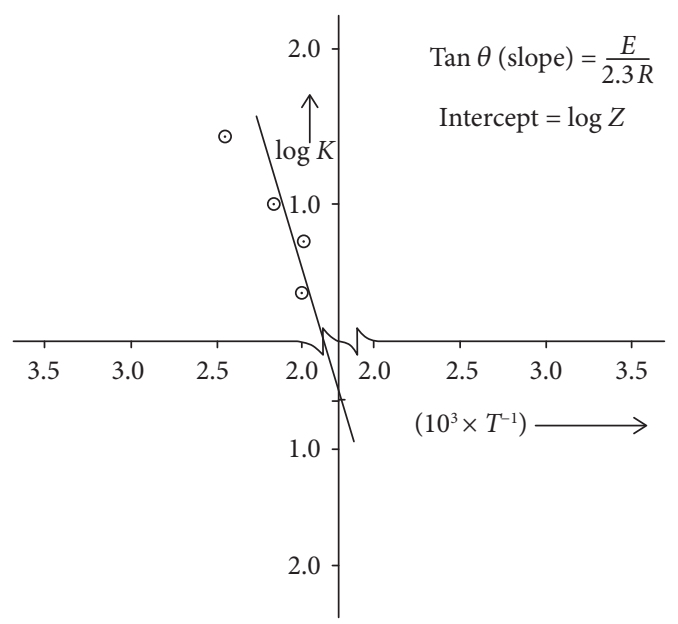

FIGURe 11: Dave and Chopra plot applying DTG traces (P II). Rxn: $\left(\mathrm{C}_{10} \mathrm{H}_{6} \mathrm{O}_{4}\right)(\mathrm{Zn})_{2} \stackrel{600^{\circ}-700^{\circ} \mathrm{C}}{\longrightarrow}\left(\mathrm{C}_{10} \mathrm{H}_{6} \mathrm{O}_{4}\right)_{2}(\mathrm{Zn})_{2}+\mathrm{C}_{10} \mathrm{H}_{6} \mathrm{O}_{4}$.

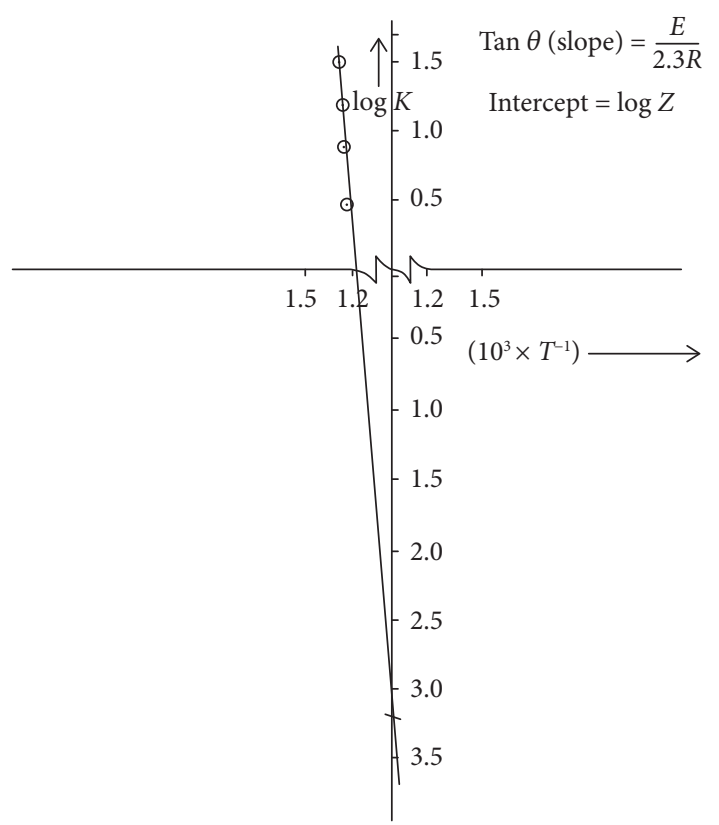

Figure 12: Dave and Chopra plot applying DTG traces (P III). Rxn: $\left[\left(\mathrm{C}_{10} \mathrm{H}_{6} \mathrm{O}_{4}\right)_{3}(\mathrm{Co})_{2} 4 \mathrm{H}_{2} \mathrm{O}\right] 3 \mathrm{H}_{2} \mathrm{O} \stackrel{100^{\circ}-169^{\circ} \mathrm{C}}{\longrightarrow}\left(\mathrm{C}_{10} \mathrm{H}_{6} \mathrm{O}_{4}\right)_{3}(\mathrm{Co})_{2}+$ $7 \mathrm{H}_{2} \mathrm{O}$.

$\begin{array}{cc}\text { TG } & \text { DTG } \\ \text { Sigmoid } & \text { Peaks } \\ 100^{\circ}-169^{\circ} \mathrm{C} & 156^{\circ} \mathrm{C}\left(33.9 \mu \mathrm{g} \mathrm{min}{ }^{-1}\right) \\ 200^{\circ}-363^{\circ} \mathrm{C} & 48^{\circ} \mathrm{C}\left(35.1 \mu \mathrm{g} \mathrm{min}^{-1}\right) \\ 500^{\circ}-600^{\circ} \mathrm{C} & 557^{\circ} \mathrm{C}\left(38.5 \mu \mathrm{g} \mathrm{min}^{-1}\right)\end{array}$

The DTA spectrum analysis led to believe "No Visible deviation" from the baseline.

The possible nonisothermal decomposition reactions identified on TG trace (Figure 7) are described as follows:

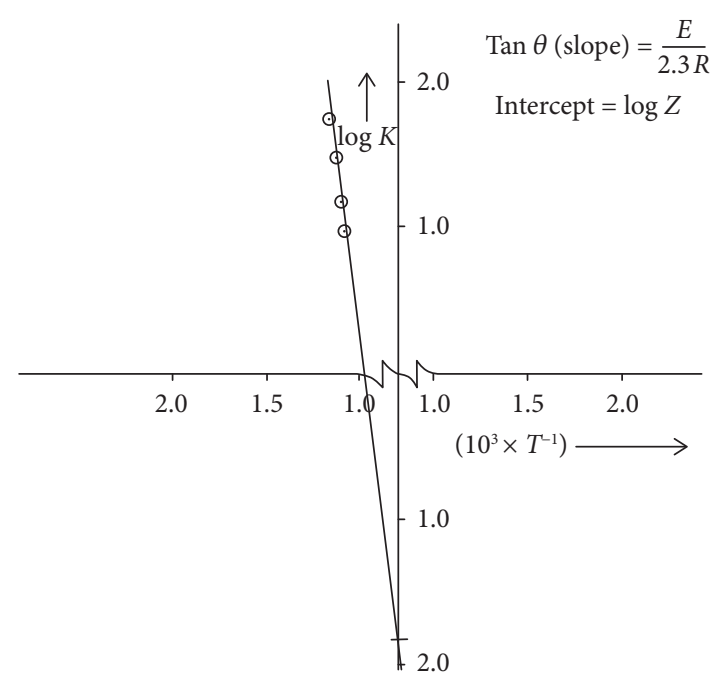

FIgure 13: Dave and Chopra plot applying DTG traces (P IV). Rxn: $\left(\mathrm{C}_{10} \mathrm{H}_{6} \mathrm{O}_{4}\right)_{3}(\mathrm{Co})_{2} \stackrel{200^{\circ}-363^{\circ} \mathrm{C}}{\longrightarrow} 3 / 2\left(\mathrm{C}_{10} \mathrm{H}_{6} \mathrm{O}_{4}\right)(\mathrm{Co})_{2}+3 / 2 \mathrm{C}_{10} \mathrm{H}_{6} \mathrm{O}_{4}$.

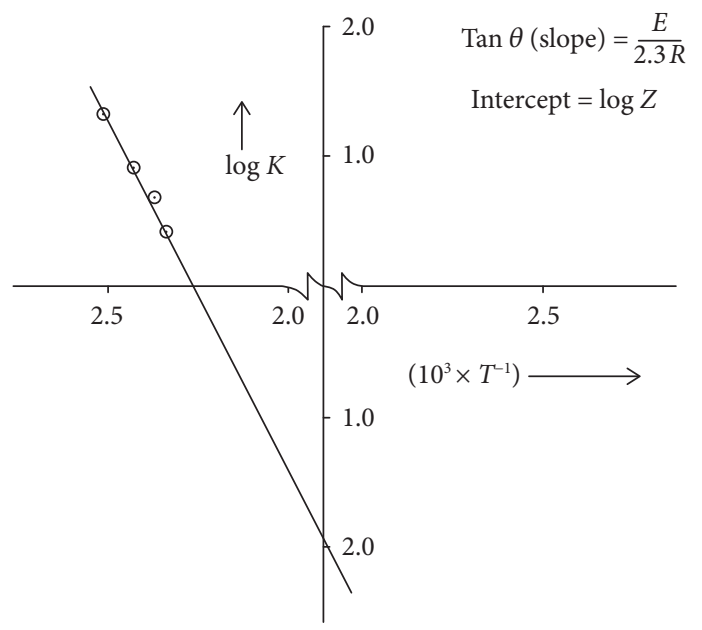

Figure 14: Dave and Chopra plot applying DTG traces (P V). Rxn: $3 / 2\left(\mathrm{C}_{10} \mathrm{H}_{6} \mathrm{O}_{4}\right)(\mathrm{Co})_{2} \stackrel{500^{\circ}-600^{\circ} \mathrm{C}}{\longrightarrow} \mathrm{Co}_{3} \mathrm{O}_{8}+3 / 2 \mathrm{C}_{10} \mathrm{H}_{6} \mathrm{O}_{4}$.

(V) $\left[\left(\mathrm{C}_{10} \mathrm{H}_{6} \mathrm{O}_{4}\right)_{3}(\mathrm{Co})_{2} 4 \mathrm{H}_{2} \mathrm{O}\right] 3 \mathrm{H}_{2} \mathrm{O} \stackrel{100^{\circ}-169^{\circ} \mathrm{C}}{\longrightarrow}\left(\mathrm{C}_{10} \mathrm{H}_{6}\right.$ $\left.\mathrm{O}_{4}\right)_{3}(\mathrm{Co})_{2}+7 \mathrm{H}_{2} \mathrm{O}$

(VI) $\left(\mathrm{C}_{10} \mathrm{H}_{6} \mathrm{O}_{4}\right)_{3}(\mathrm{Co})_{2} \stackrel{200^{\circ}-363^{\circ} \mathrm{C}}{\longrightarrow} 3 / 2\left(\mathrm{C}_{10} \mathrm{H}_{6} \mathrm{O}_{4}\right)_{3}(\mathrm{Co})_{2}+$ $3 / 2 \mathrm{C}_{10} \mathrm{H}_{6} \mathrm{O}_{4}$

(VII) $3 / 2\left(\mathrm{C}_{10} \mathrm{H}_{6} \mathrm{O}_{4}\right)(\mathrm{Co})_{2} \stackrel{500^{\circ}-600^{\circ} \mathrm{C}}{\longrightarrow} \mathrm{Co}_{3} \mathrm{O}_{8}+3 / 2 \mathrm{C}_{10} \quad \mathrm{H}_{6} \mathrm{O}_{4}$

3.3. Polymetallic Chelate of Naphthazarin with $\mathrm{Ni}$ (II). Composition: $\left[\left(\mathrm{C}_{10} \mathrm{H}_{6} \mathrm{O}_{4}\right)_{3}(\mathrm{Ni})_{2} 4 \mathrm{H}_{2} \mathrm{O}\right] 1 \frac{1}{2} 2 \mathrm{H}_{2} \mathrm{O}$.

3.3.1. Proposed Structure. The $\left[\left(\mathrm{C}_{10} \mathrm{H}_{6} \mathrm{O}_{4}\right)_{3} \quad(\mathrm{Ni})_{2} .4 \mathrm{H}_{2} \mathrm{O}\right]$ $1 \frac{1}{2} \mathrm{H}_{2} \mathrm{O}$ exhibited thermally the initial plateau, ambience to $99.9^{\circ} \mathrm{C}$, showing the structural stability, which showed the 


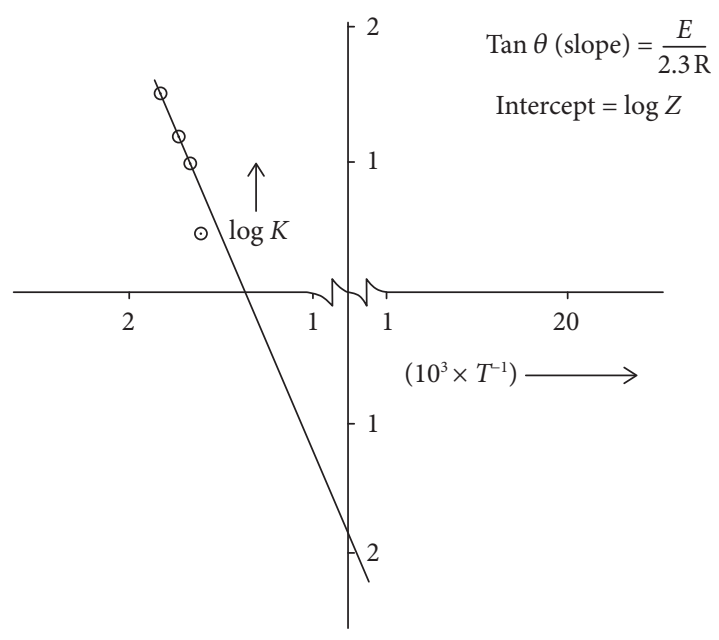

Figure 15: Dave and Chopra plot applying DTG traces (P VI). Rxn: $\left[\left(\mathrm{C}_{10} \mathrm{H}_{6} \mathrm{O}_{4}\right)_{3}(\mathrm{Ni})_{2} 4 \mathrm{H}_{2} \mathrm{O}\right] 11 / 2 \mathrm{H}_{2} \mathrm{O} \stackrel{99.9^{\circ}-136^{\circ} \mathrm{C}}{\longrightarrow}\left(\mathrm{C}_{10} \mathrm{H}_{6} \mathrm{O}_{4}\right)_{3}(\mathrm{Ni})_{2}+$ $11 / 2 \mathrm{H}_{2} \mathrm{O}$.

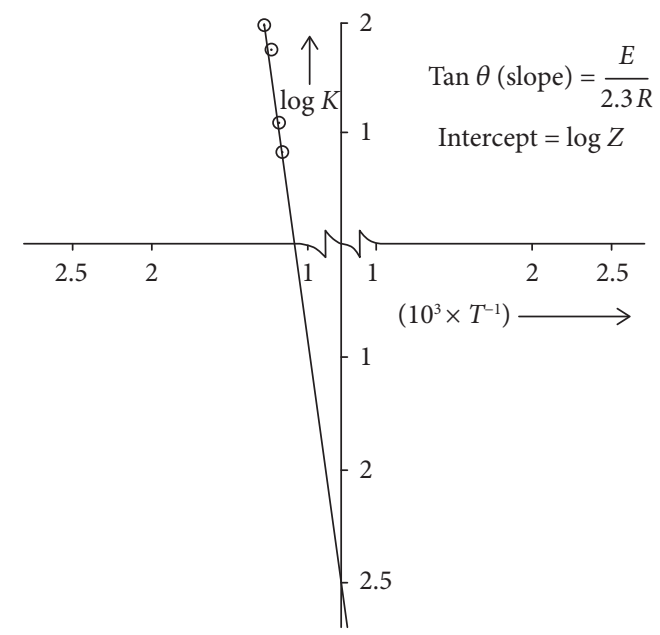

Figure 16: Dave and Chopra plot applying DTG traces (P VII). Rxn: $\left(\mathrm{C}_{10} \mathrm{H}_{6} \mathrm{O}_{4}\right)_{3}(\mathrm{Ni})_{2} \stackrel{300^{\circ}-365^{\circ} \mathrm{C}}{\longrightarrow} 5 / 4\left(\mathrm{C}_{10} \mathrm{H}_{6} \mathrm{O}_{4}\right)_{3}(\mathrm{Ni})_{2}+13 / 4 \mathrm{C}_{10} \mathrm{H}_{6} \mathrm{O}_{4}$.

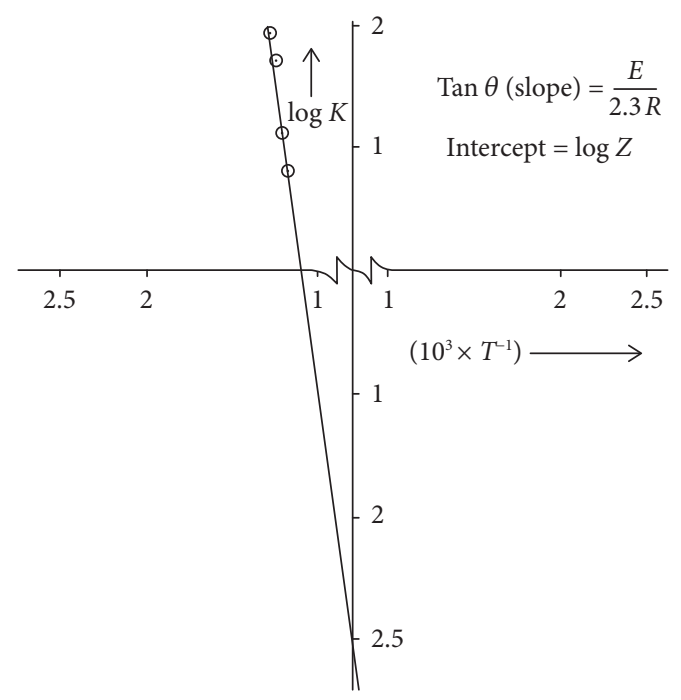

Figure 17: Dave and Chopra plot applying DTG traces (P VIII). Rxn: $5 / 4\left(\mathrm{C}_{10} \mathrm{H}_{6} \mathrm{O}_{4}\right)_{3}(\mathrm{Ni})_{2} \stackrel{500^{\circ}-600^{\circ} \mathrm{C}}{\longrightarrow} \mathrm{NiO}+5 / 4 \mathrm{C}_{10} \mathrm{H}_{6} \mathrm{O}_{4}$.

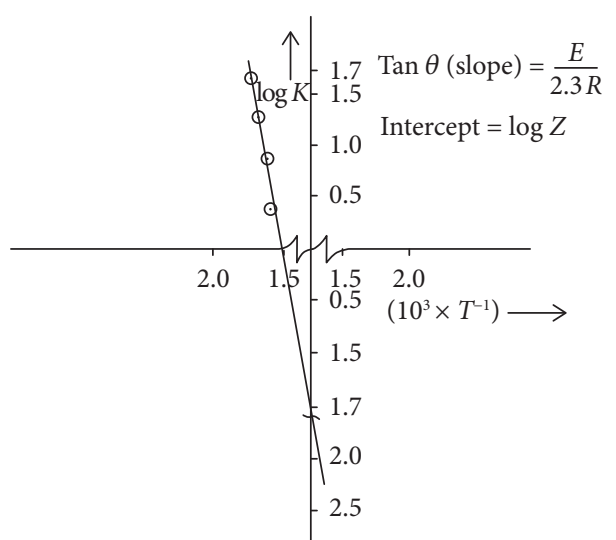

Figure 18: Dave and Chopra plot applying DTG traces (P IX). Rxn: $\left(\mathrm{C}_{10} \mathrm{H}_{6} \mathrm{O}_{4}\right)_{3}(\mathrm{Cu})_{2} 4 \mathrm{H}_{2} \mathrm{O} \stackrel{26.5^{\circ}-71^{\circ} \mathrm{C}}{\longrightarrow}\left(\mathrm{C}_{10} \mathrm{H}_{6} \mathrm{O}_{4}\right)_{3}(\mathrm{Cu})_{2}+4 \mathrm{H}_{2} \mathrm{O}$.

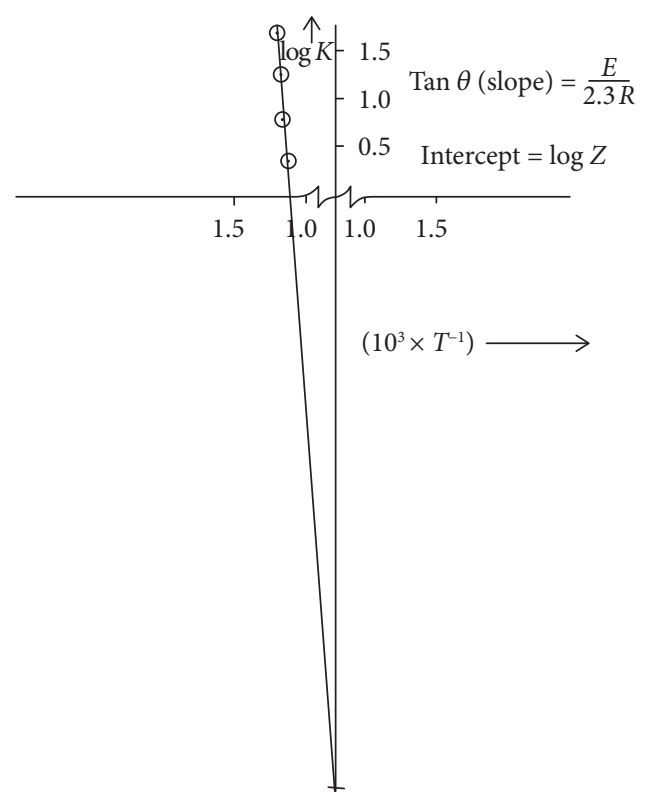

Figure 19: Dave and Chopra plot applying DTG traces (P X). Rxn: $\left(\mathrm{C}_{10} \mathrm{H}_{6} \mathrm{O}_{4}\right)_{3}(\mathrm{Cu})_{2} \stackrel{300^{\circ}-400^{\circ} \mathrm{C}}{\longrightarrow}\left(\mathrm{C}_{10} \mathrm{H}_{6} \mathrm{O}_{4}\right)_{2}(\mathrm{Cu})_{2}+\mathrm{C}_{10} \mathrm{H}_{6} \mathrm{O}_{4}$.

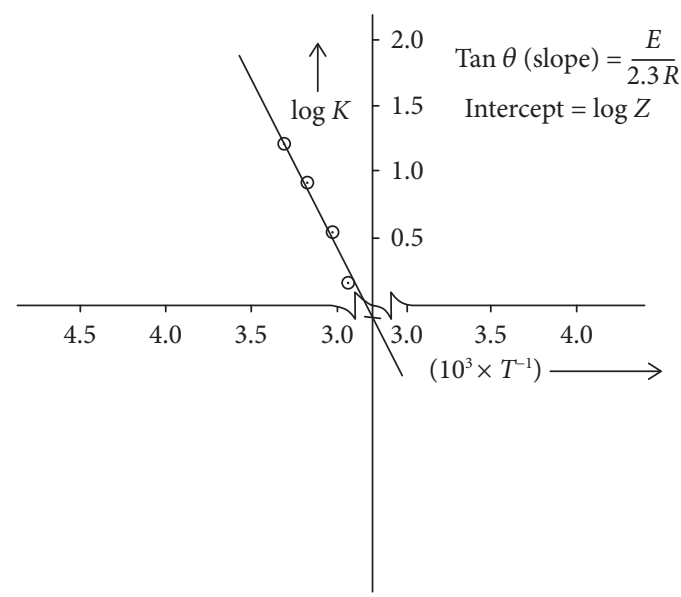

Figure 20: Dave and Chopra plot applying DTG traces (P XI). 


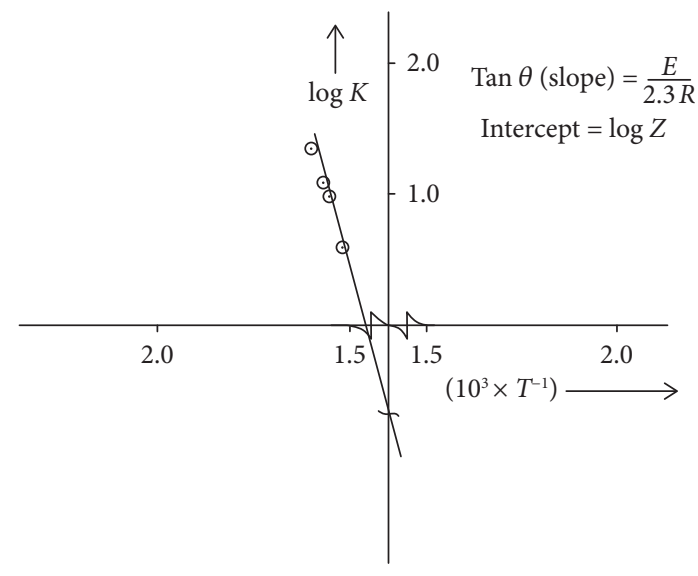

FIGURE 21: Dave and Chopra plot applying DTG traces (P XII).

degeneration with the rising temperature above $99.9^{\circ} \mathrm{C}$ (pdt: $99.9^{\circ} \mathrm{C}$ ). The structural degeneration ended at $136^{\circ} \mathrm{C}$ with the departure of coordinated and lattice water molecules, ending at the intermediate composition, $\left(\mathrm{C}_{10} \mathrm{H}_{6} \mathrm{O}_{4}\right)_{3}(\mathrm{Ni})_{2}$, showing weight constancy in temperature range $136^{\circ} \mathrm{C}-300^{\circ} \mathrm{C}$. Further supply of energy to $\left(\mathrm{C}_{10} \mathrm{H}_{6} \mathrm{O}_{4}\right)_{3}(\mathrm{Ni})_{2}$ caused more degeneration in the structural design of the intermediate composition in the temperature of $300^{\circ}-365^{\circ} \mathrm{C}$, leaving behind $5 / 4$ $\left(\mathrm{C}_{10} \mathrm{H}_{6} \mathrm{O}_{4}\right)(\mathrm{Ni})_{2}$ with the loss of $13 / 4 \mathrm{C}_{10} \mathrm{H}_{6} \mathrm{O}_{4}$ molecule. The 5/ $4\left(\mathrm{C}_{10} \mathrm{H}_{6} \mathrm{O}_{4}\right) \quad(\mathrm{Ni})_{2}$ intermediate composition (stable at $365^{\circ}-500^{\circ} \mathrm{C}$ ) could not tolerate the impact structurally at the temperature above $500^{\circ} \mathrm{C}$. The residual organics attached with the metal was lost in the temperature range of $500^{\circ}-600^{\circ} \mathrm{C}$, leaving finally the mass with correspondence to $\mathrm{NiO}$.

The peaks recorded on DTG traces $\left(132^{\circ} \mathrm{C}(4.12 \mu \mathrm{g} / \mathrm{min})\right.$, $350^{\circ} \mathrm{C}(7.7 \mu \mathrm{g} / \mathrm{min})$, and $\left.577^{\circ} \mathrm{C}(8.4 \mu \mathrm{g} / \mathrm{min})\right)$ had the clear tally with the corresponding sigmoids on TG traces $\left(99.9^{\circ}-136^{\circ} \mathrm{C}, 300^{\circ}-365^{\circ} \mathrm{C}\right.$, and $500^{\circ}-600^{\circ} \mathrm{C}$ ).

The possible nonisothermal decomposition reactions identified on TG trace (Figure 8) are described as follows:

$$
\begin{aligned}
& \text { (VIII) } {\left[\left(\mathrm{C}_{10} \mathrm{H}_{6} \mathrm{O}_{4}\right)_{3}(\mathrm{Ni})_{2} 4 \mathrm{H}_{2} \mathrm{O}\right] 11 / 2 \mathrm{H}_{2} \mathrm{O} \stackrel{99.9^{\circ}-136^{\circ} \mathrm{C}}{\longrightarrow} } \\
&\left(\mathrm{C}_{10} \mathrm{H}_{6} \mathrm{O}_{4}\right)_{3}(\mathrm{Ni})_{2}+51 / 2 \mathrm{H}_{2} \mathrm{O} \\
& \text { (IX) }\left(\mathrm{C}_{10} \mathrm{H}_{6} \mathrm{O}_{4}\right)_{3}(\mathrm{Ni})_{2} \stackrel{300^{\circ}-365^{\circ} \mathrm{C}}{\longrightarrow} 7 / 4\left(\mathrm{C}_{10} \mathrm{H}_{6} \mathrm{O}_{4}\right)_{3}(\mathrm{Ni})_{2}+ \\
& 11 / 4 \mathrm{C}_{10} \mathrm{H}_{6} \mathrm{O}_{4} \\
& \text { (X) } 7 / 4\left(\mathrm{C}_{10} \mathrm{H}_{6} \mathrm{O}_{4}\right)_{3}(\mathrm{Ni})_{2} \stackrel{500^{\circ}-600^{\circ} \mathrm{C}}{\longrightarrow} \mathrm{NiO}+7 / 4 \mathrm{C}_{10} \mathrm{H}_{6} \mathrm{O}_{4}
\end{aligned}
$$

The analytical data on the pyrolysis journey on the polymetallic chelate of naphthazarin with nickel as shown by TG mapping are given in Table 3.

\subsection{Polymetallic Chelate of Naphthazarin with $\mathrm{Cu}$ (II). Composition: $\left[\left(\mathrm{C}_{10} \mathrm{H}_{6} \mathrm{O}_{4}\right)_{3}(\mathrm{Cu})_{2}\right] 4 \mathrm{H}_{2} \mathrm{O}$.}

3.4.1. Proposed Structure. The spread of temperature range ambience at $800^{\circ} \mathrm{C}$, the instrumental condition could not cause complete combustion, that is, the departure of $03 \mathrm{C}_{10} \mathrm{H}_{6} \mathrm{O}_{4}$ molecules and 04 lattice water molecules from the parent polymetallic chelate, $\left[\left(\mathrm{C}_{10} \mathrm{H}_{6} \mathrm{O}_{4}\right)_{3}(\mathrm{Cu})_{2}\right] 4 \mathrm{H}_{2} \mathrm{O}$, with the initial range ambience, $26.5^{\circ} \mathrm{C}$, as the initial thermal stability of the metal chelate. The incremental rise
TABLE 5: The kinetics data on different solid state reactions.

\begin{tabular}{lccc}
\hline Reaction & E $\left(\mathrm{kcal}_{\mathrm{mol}}^{-1}\right)$ & $\mathrm{Z}$ & DTG peak \\
\hline I & 2.25 & $2.8 \times 10^{-2}$ & P I (Figures 3-6) \\
II & 1.75 & $47.8 \times 10^{-2}$ & P II (Figures 3-6) \\
III & 1.6 & $5.4 \times 10^{-4}$ & P III (Figures 3-6) \\
IV & 4.75 & $15.1 \times 10^{-3}$ & P IV (Figures 3-6) \\
V & 5.5 & $10.9 \times 10^{-3}$ & P V (Figures 3-7) \\
VI & 16 & $15.8 \times 10^{-3}$ & P VI (Figures 3-7) \\
VII & 8.0 & $1.0 \times 10^{-3}$ & P VII (Figures 3-7) \\
VIII & 6.0 & $3.63 \times 10^{-3}$ & P VIII (Figures 3-8) \\
IX & 6.6 & $2.5 \times 10^{-2}$ & P IX (Figures 3-8) \\
X & 22 & $8.7 \times 10^{-0.7}$ & P X (Figures 3-8) \\
XI & 2.0 & $79.4 \times 10^{-2}$ & P XI (Figures 3-9) \\
XII & 0.8 & $60.5 \times 10^{-2}$ & P XII (Figures 3-9) \\
XIII & 6.1 & $36.3 \times 10^{-2}$ & P XIII (Figures 3-9) \\
\hline
\end{tabular}

of temperature till $71^{\circ} \mathrm{C}$ (pdt: $71^{\circ} \mathrm{C}$ ) caused $04 \mathrm{H}_{2} \mathrm{O}$ lattice water to depart, leaving behind $\left[\left(\mathrm{C}_{10} \mathrm{H}_{6} \mathrm{O}_{4}\right)_{3}(\mathrm{Cu})_{2}\right]$, the structural intermediate composition, showing weight constancy between $71^{\circ} \mathrm{C}$ and $300^{\circ} \mathrm{C}$. The sigmoid II began at $300^{\circ} \mathrm{C}$ and ended at $400^{\circ} \mathrm{C}$ agreeing analytically with loss of $\mathrm{C}_{10} \mathrm{H}_{6} \mathrm{O}_{4}$ molecule. The plateau $400^{\circ}-500^{\circ} \mathrm{C}$ had been found analytically true with the $\left[\left(\mathrm{C}_{10} \mathrm{H}_{6} \mathrm{O}_{4}\right)_{3}(\mathrm{Cu})_{2}\right]$ composition, which assumed structural degeneration when the temperature began to increase slowly above $500^{\circ} \mathrm{C}$. The departure of $1 \frac{1}{2} \mathrm{C}_{10} \mathrm{H}_{6} \mathrm{O}_{4}$ molecules ended at $545^{\circ} \mathrm{C}$ leaving behind $1 / 2\left(\mathrm{C}_{10} \mathrm{H}_{6} \mathrm{O}_{4}\right)(\mathrm{Cu})_{2}$, agreeably true to the last plateau at $545^{\circ}-800^{\circ} \mathrm{C}$. The last plateau $\left(545^{\circ} \mathrm{C}-800^{\circ} \mathrm{C}\right)$, beyond which the pyrolysis could not be extended $\left(780^{\circ} \mathrm{C}\right)$, clearly indicated the incomplete combustion of the metal chelate in reference under the applied instrumental conditions. The 03 sigmoids traces on the TG spectrum had the correspondence to the peaks registered on DTG trace of the metal chelate. The instrument registered 2nd peak contiguous to the 3rd peak on DTG trace showing near correspondence to the sigmoid tracing on TG traces (Figure 9).

$\begin{array}{cc}\text { TG } & \text { DTG } \\ \text { Sigmoid } & \text { Peaks } \\ 26.5^{\circ}-71^{\circ} \mathrm{C} & 68^{\circ} \mathrm{C}(1.09 \mu \mathrm{g} / \mathrm{min}) \\ 300^{\circ}-400^{\circ} \mathrm{C} & 350^{\circ} \mathrm{C} \\ 500^{\circ}-545^{\circ} \mathrm{C} & 533^{\circ} \mathrm{C}(1.75 \mu \mathrm{g} / \mathrm{min})\end{array}$

The possible nonisothermal decomposition reactions identified on TG trace (Figures 3-8) are described as follows:

$$
\begin{aligned}
\text { (XI) } & \left(\mathrm{C}_{10} \mathrm{H}_{6} \mathrm{O}_{4}\right)_{3} \quad(\mathrm{Cu})_{2} 4 \mathrm{H}_{2} \mathrm{O}^{26.5^{\circ}-70^{\circ} \mathrm{C}} \longrightarrow\left(\mathrm{C}_{10} \mathrm{H}_{6} \mathrm{O}_{4}\right)_{3} \\
& (\mathrm{Cu})_{2}+4 \mathrm{H}_{2} \mathrm{O} \\
\text { (XII) } & \left(\mathrm{C}_{10} \mathrm{H}_{6} \mathrm{O}_{4}\right)_{3}(\mathrm{Cu})_{2} \stackrel{300^{\circ}-400^{\circ} \mathrm{C}}{\longrightarrow}\left(\mathrm{C}_{10} \mathrm{H}_{6} \mathrm{O}_{4}\right)_{3} \quad(\mathrm{Cu})_{2}+ \\
& \mathrm{C}_{10} \mathrm{H}_{6} \mathrm{O}_{4} \\
(\mathrm{XIII}) & \left(\mathrm{C}_{10} \mathrm{H}_{6} \mathrm{O}_{4}\right)_{3}(\mathrm{Cu})_{2} \stackrel{545^{\circ} \mathrm{C}}{\longrightarrow} 1 / 2\left(\mathrm{C}_{10} \mathrm{H}_{6} \mathrm{O}_{4}\right)_{3}(\mathrm{Cu})_{2}+ \\
& 3 / 2\left(\mathrm{C}_{10} \mathrm{H}_{6} \mathrm{O}_{4}\right)
\end{aligned}
$$

The analytical data on the pyrolysis journey on the polymetallic chelate of naphthazarin with copper as shown by TG mapping are given in Table 4 . 
3.5. Kinetics and Solid State Reactions. The I-XIII solid state reactions identified on the TG traces with correspondence to DTG peaks consequent to the nonisothermal decomposition of polymetallic chelates of the naphthazarin with $\mathrm{Zn}$ (II), $\mathrm{Co}$ (II), $\mathrm{Ni}$ (II), and $\mathrm{Cu}$ (II) over the temperature range ambient at $800^{\circ} \mathrm{C}$ have been studied kinetically following the Dave and Chopra method as these solid state reactions exhibited their resemblance with the Freeman recommended reaction for kinetic studies.

$\mathrm{A}(\mathrm{s}) \longrightarrow \mathrm{B}(\mathrm{s})+\mathrm{C}(\mathrm{g}) \uparrow$.

The sigmoid sandwiched between two plateaus represents a solid state reaction (Scheme 2).

For each solid state reaction, the terms $A, a$, and $-\mathrm{d} x / \mathrm{d} t$ at various $T$ values employ DTG traces corresponding to sigmoids on TG trace. The plot of log $k$ (where $k=(-d x / d t) /(A-a))$ against the reciprocal of absolute temperature $(T)$ gave a straight line relationship justifying the assumption of order of reaction $(n)$ as one.

Figures 10-21 represent the Dave and Chopra plots for $n=1$ for different solid state reactions (I-XIII), giving slope $(\tan \theta)$ as $E / 2.3 R$ and intercept as $\log Z$. The characteristics terms $E$ and $Z$ for solid state reactions (I-XIII) are tabulated in Table 5.

It is an established fact that the velocity rate increases with the rise of temperature according to collision theory for reactions, justifying more collisions among the involved molecules. This means that the $Z$ (frequency factor or collisions number) value rises with more collisions among the molecules describing the reactions involved as fast reaction, but the lower values of $Z$ may help conclude the reaction under study as slow in nature. The kinetic data showed the very low value of $Z$ for each of the solid state reaction in reference (Table 5), concluding on the solid state reaction (I-XIII) (the nonisothermal decomposition of polymetallic chelate of $\mathrm{Zn}(\mathrm{II}), \mathrm{Co}(\mathrm{II}), \mathrm{Ni}(\mathrm{II})$, and $\mathrm{Cu}(\mathrm{II})$ as slow reactions).

The DTG traces on the polymetallic chelates of naphthazarin with $\mathrm{Zn}$ (II), $\mathrm{Co}$ (II), $\mathrm{Ni}$ (II), and $\mathrm{Cu}$ (II) are shown in Figures 6-9, respectively.

\section{Conclusion}

The pyrolysis spectrum of each of the polymetal chelates exhibiting plateaus and sigmoids with correspondence on the DTG traces but with no responses on DTA traces have led us to conclude on the structures of the polymetal chelates tentatively.

The unidentified nonisothermal decomposition of polymetallic chelates of naphthazarin with resemblance to $\mathrm{A}(\mathrm{s}) \longrightarrow \mathrm{B}(\mathrm{s})+\mathrm{C}(\mathrm{g})$ (reaction: sigmoid flanked by plateaus on the trace) has been studied kinetically applying the Dave and Chopra method and DTG traces. The solid state reactions described followed first order kinetics. The kinetic data showed the very low value of $Z$ for each of the solid state reaction in reference, concluding on the solid state reactions (the nonisothermal decomposition of polymetallic chelate of $\mathrm{Zn}$ (II), Co (II), Ni (II), and Cu (II) as slow reactions).

\section{Data Availability}

The data used to support the findings of this study are available from the corresponding author upon request.

\section{Conflicts of Interest}

The authors declare that there are no conflicts of interest.

\section{Acknowledgments}

The authors are thankful to PMU, Saudi Arabia, for allowing them to work on the project in reference.

\section{References}

[1] M. Borrel and R. Pâris, "Analyse thermogravimétrique des principaux oxinates métalliques," Analytica Chimica Acta, vol. 4, pp. 267-285, 1950.

[2] M. Borrel and R. Pâris, "Analyse thermogravimétrique de quelques méthyl-2-oxinates métalliques," Analytica Chimica Acta, vol. 5, pp. 573-583, 1951.

[3] M. Borrel and R. A. Pâris, "Domaines de précipitation et produits de solubilité de quelques oxinates et méthyloxinates métalliques," Analytica Chimica Acta, vol. 6, pp. 389-399, 1952.

[4] R. G. Charles and A. Langer, "Heat stabilities and volatilities of some metal chelates derived from 8-hydroxyquinoline," The Journal of Physical Chemistry, vol. 63, no. 4, pp. 603-605, 1959.

[5] G. D. Ascenzo, U. Biader Ceipidor, and G. De Angelis, "Thermal properties of some compounds of lutidinic acid with divalent metals," Analytica Chimica Acta, vol. 58, no. 1, pp. 175-182, 1972.

[6] R. H. Gore and W. W. Wendlandt, "Thermal dehydration of some 8-quinolinol chelate hydrates," Analytica Chimica Acta, vol. 52, no. 1, pp. 83-89, 1970.

[7] G. J. Palenik, "The structure of coordination compounds. II. The crystal and molecular structure of the $\beta$ form of anhydrous copper 8-hydroxyquinolinate," Acta Crystallographica, vol. 17, no. 6, pp. 687-695, 1964.

[8] R. C. Hoy and R. H. Morriss, "The crystal structure of the $\alpha$ form of anhydrous copper 8-hydroxyquinolinate," Acta Crystallographica, vol. 22, no. 4, pp. 476-482, 1967.

[9] E. Suito and E. Sekido, "The dimorphism and the crystal habits of copper-oxinate precipitates," Proceedings of the Japan Academy, vol. 33, no. 4, pp. 196-200, 1957.

[10] L. L. Merritt Jr., R. T. Cady, and B. W. Mundy, "X-ray structure determinations," Analytical Chemistry, vol. 25, no. 5, pp. 718-721, 1953.

[11] G. J. Palenik, "The structure of coordination compounds. III. A refinement of the structure of zinc 8-hydroxyquinolinate dihydrate," Acta Crystallographica, vol. 17, no. 6, pp. 696-700, 1964.

[12] W. Andruchow Jr. and R. D. Archer, "Molybdenum(V) oxocomplexes of 8-quinolinol," Journal of Inorganic and Nuclear Chemistry, vol. 34, no. 10, pp. 3185-3195, 1972.

[13] R. G. Charles, "Solid state synthesis of a new compound $\mathrm{Cu}$ (HQ) $\mathrm{Cl} 2$ and its formation reaction," Spectrochimica Acta, vol. 8, p. 1, 1956.

[14] J. E. Tackett and D. T. Sawyer, "Properties and infrared spectra in the potassium bromide region of 8-quinolinol and its metal chelates," Inorganic Chemistry, vol. 3, no. 5, pp. 692-696, 1964. 
[15] R. Magee and L. Gordon, "The infrared spectra of chelate compounds-I A study of some metal chelate compounds of 8 hydroxyquinoline in the region 625 to $5000 \mathrm{~cm}^{-1}$," Talanta, vol. 10 , no. 8, pp. 851-859, 1963.

[16] R. Larson and O. Eskilsson, "Preparation and thermal decomposition of copper (II), zinc (II) and cadmium (II) chelates with 8-hydroxyquinoline," Acta Chemica Scandinavica, vol. 22, p. 1067, 1968.

[17] N. Ohkaku and K. Nakamoto, "Dioxouranium (VI) complexes of $\mathrm{N}$-(orthovanillidene)-anthranilic acid and $\mathrm{N}$ (vanillidene)-anthranilic acid," Inorganic Chemicals, vol. 10, p. 798, 1971.

[18] E. J. Corey and J. C. Bailar Jr., "The stereochemistry of complex inorganic compounds. XXII. Stereospecific effects in complex ions," Journal of the American Chemical Society, vol. 81, p. 152,1959

[19] J. M. Sánchez-Calvo, G. R. Barbero, G. Guerrero-Vásquez et al., "Synthesis, antibacterial and antifungal activities of naphthoquinone derivatives: a structure-activity relationship study," Medicinal Chemistry Research, vol. 25, no. 6, pp. 1274-1285, 2016.

[20] D. Aminin and S. Polonik, "1,4-Naphthoquinones: some biological properties and application," Chemical and Pharmaceutical Bulletin, vol. 68, no. 1, pp. 46-57, 2020.

[21] T. Kobayashi, M. Haruta, K. Sasagawa et al., "Optical communication with brain cells by means of an implanted duplex micro-device with optogenetics and Ca2+ fluoroimaging," Science Reports, vol. 6, 2016.

[22] Y. Hou, E. A. Vasileva, A. Carne, M. McConnell, A. El-Din et al., "Naphthoquinones of the spinochrome class: occurrence, isolation, biosynthesis and biomedical applications," RSC Advances, vol. 8, no. 57, pp. 32637-32650, 2018.

[23] K. W. Wellington, N. I. Kolesnikova, V. Hlatshwayo, S. T. Saha, M. Kaur, and L. R. Motadi, "Anticancer activity, apoptosis and a structure-activity analysis of a series of 1,4naphthoquinone-2,3-bis-sulfides," Investigational New Drugs, vol. 38, no. 2, pp. 274-286, 2019.

[24] A. A. Aly, E. M. El-Sheref, M. E. M. Bakheet et al., "Synthesis of novel 1,2-bis-quinolinyl-1,4-naphthoquinones: ERK2 inhibition, cytotoxicity and molecular docking studies," Bioorganic Chemistry, vol. 81, pp. 700-712, 2018.

[25] M. Badolato, G. Carullo, M. C. Caroleo, E. Cione, F. Aiello, and F. Manetti, "Discovery of 1,4-naphthoquinones as a new class of antiproliferative agents targeting GPR55," ACS Medicinal Chemistry Letters, vol. 10, no. 4, pp. 402-406, 2019.

[26] S. A. Dyshlovoy, D. N. Pelageev, J. Hauschild et al., "Successful targeting of the warburg effect in prostate cancer by glucoseconjugated 1,4-naphthoquinones," Cancers, vol. 11, no. 11, p. 1690, 2019.

[27] E. S. Freeman and B. Carroll, "The application of thermoanalytical techniques to reaction kinetics: the thermogravimetric evaluation of the kinetics of the decomposition of calcium oxalate monohydrate," The Journal of Physical Chemistry, vol. 6-2, no. 4, pp. 394-397, 1958.

[28] N. G. Dave and S. K. Chopra, "A method for the evaluation of kinetics of solid state reactions from thermogravimetric curves," Journal of Physical Chemistry, vol. 48, no. 5-6, pp. 257-266, 1966. 